\title{
Semigroup-theoretic approach to diffusion in thin layers separated by semi-permeable membranes
}

\author{
ADAM BOBROWSKID
}

Abstract. Using techniques of the theory of semigroups of linear operators, we study the question of approximating solutions to equations governing diffusion in thin layers separated by a semi-permeable membrane. We show that as thickness of the layers converges to 0 , the solutions, which by nature are functions of 3 variables, gradually lose dependence on the vertical variable and thus may be regarded as functions of 2 variables. The limit equation describes diffusion on the lower and upper sides of a two-dimensional surface (the membrane) with jumps from one side to the other. The latter possibility is expressed as an additional term in the generator of the limit semigroup, and this term is built from permeability coefficients of the membrane featuring in the transmission conditions of the approximating equations (i.e., in the description of the domains of the generators of the approximating semigroups). We prove this convergence result in the spaces of square integrable and continuous functions, and study the way the choice of transmission conditions influences the limit.

\section{Introduction}

\subsection{Main results}

The paper is devoted to a semigroup-theoretic approach to the problem of approximating solutions to an equation modeling diffusion in two thin 3D layers separated by a semi-permeable membrane: particles diffusing in the upper layer may, via a stochastic mechanism, filter through the membrane to the lower layer and continue their chaotic movement there, and vice versa. To this end, we study the reaction-diffusion equation

$$
\partial_{t} u=\Delta_{3 D} u+F(u)
$$

where $\Delta_{3 D}$ is a $3 D$ Laplace operator, and $F$ is a Lipschitz continuous forcing (reaction) term, considered in two layers of thickness $\varepsilon$, equipped with boundary and transmission conditions (see (2.3) and (3.1), further down) that describe in particular the way the membrane works. As a starting point, we prove appropriate generation theorems in the

Mathematics Subject Classification: 35K57, 47D06, 35B25, 35K58

Keywords: Semigroups of operators, Semilinear equations, Reaction-diffusion equations, Irregular convergence, Singular perturbations, Boundary and Transmission conditions, Thin layers.

This research is supported by National Science Center (Poland) Grant 2017/25/B/ST1/01804. 
spaces of square integrable and continuous functions, respectively (thus establishing existence and uniqueness of mild solutions of the equation). Next, we show that, as $\varepsilon \rightarrow 0$, the approximating processes resemble more and more 2D Brownian motions on the upper and lower sides of the membrane. Remarkably, the limit process allows also jumps from one side to the other: this possibility is the limit equivalent of the mechanism of filtering through the membrane in the approximating process. More specifically, as $\varepsilon \rightarrow 0$ and as looked upon through a magnifying glass (see below), solutions of (1.1) become more and more flat in the vertical direction (but still differ in the lower and upper layers) and thus may be identified with pairs $\left(u^{-}, u^{+}\right)$of functions of two variables, defined on the lower and upper sides of the membrane. The limit dynamics is then governed by the following system:

$$
\partial_{t}\left(\begin{array}{l}
u^{-} \\
u^{+}
\end{array}\right)=\left[\left(\begin{array}{cc}
\Delta_{2 D} & 0 \\
0 & \Delta_{2 D}
\end{array}\right)+\left(\begin{array}{cc}
-\alpha & \alpha \\
\beta & -\beta
\end{array}\right)\right]\left(\begin{array}{l}
u^{-} \\
u^{+}
\end{array}\right)+\left(\begin{array}{l}
F\left(u^{-}\right)-c^{-} u^{-} \\
F\left(u^{+}\right)-c^{+} u^{+}
\end{array}\right)
$$

where $\Delta_{2 D}$ is a 2D Laplace operator. More interestingly, $\alpha$ and $\beta$ are functions that describe permeability of the membrane in the approximating processes, featuring in the transmission conditions of the approximating equations (see (2.3) and (3.1) again). Functions $c^{-}$and $c^{+}$play a similar role: they come from the Robin boundary conditions in the approximating processes (see (2.2)), describing partial loss of particles touching lower and upper boundaries of the layers. Thus, our theorem extends the findings of $[23,24]$ by saying that

in the thin layer approximation, both boundary and transmission conditions become integral parts of the master equation.

This, in fact, is the main phenomenon to which this paper is devoted, and a common thread for the series of articles [19,23,24] (see also below); paper [19] is a similar study in two dimensions.

It is also worth noting that processes described by (1.2) are closely related to piecewise deterministic Markov processes of Davis [31-33,78], random evolutions of Griego and Hersh [36,44,45,70] and to randomly switching diffusions [49,85,86]; for a semigroup-theoretic context see $[12,17]$. The proposition that such processes are obtained by the thin layer approximation is of interest in itself, and provides a link between notions of jump intensities and permeability coefficients, i.e., shows again that transmission conditions and terms describing jumps play complementary roles (cf. Remark 2.1 in [21], and the discussion in Sect. 6 of [19]).

As already mentioned, we prove two variants of (generation and) approximation results, as summarized in Theorems 1 and 3, respectively. Both convergence results say that (1.2) is a limit form of (1.1) but they nevertheless differ in a couple of ways. First of all, but somewhat superficially, Theorem 1 is devoted to semigroups in the space of square integrable functions, whereas Theorem 3 subsumes our analysis in the space of continuous functions. More intrinsically, however, in the former theorem the membrane separating two thin layers is nonadhesive, whereas in the latter it is 
sticky, and in fact by varying certain coefficients we may control its stickiness from completely nonadhesive to completely adhesive. On the other hand, in Theorem 1 permeability coefficients are bounded, measurable functions on the membrane, and thus, permeability may vary from region to region, whereas in Theorem 3 these permeability coefficients, for technical reasons, need to be constant. Nevertheless, the limit dynamics in both cases is (almost) the same, that is, our approximation theorem is robust to changing the mechanism of filtering through the membrane.

As a matter of fact, a comparison of Theorems 1 and 3 gives a deeper insight into the way transmission conditions influence the limit master equation, and to study this influence is the second object of our analysis. To explain the result, we note first that, intuitively, the reason why (1.1) has the limit form (1.2) is that a thin layer approximation is, when looked upon through a magnifying glass, equivalent to studying fast diffusion in the direction where the layer is thin, and such diffusion averages out, or flattens, solutions in this direction (cf. [16]). A closer inspection reveals, however, that different transmission conditions, which correspond to different ways particles may filter through the separating membrane, lead to different averaging mechanisms. In particular, even though the limit equation (1.2) is in both cases the same, the initial condition associated with it is not (see the end of Sect. 3 for details).

\subsection{Motivation}

Our research is motivated by the study of signaling pathways in eukaryotes. Kinases, special enzymes, play a key role in a number of signal-transmitting processes, and their activity may often be modeled by reaction-diffusion equations $[47,48,54]$. It has been observed that geometry of the cell has significant impact on the behavior of solutions of such equations and thus on the process of signal transduction. For example, so-called $B$ lymphocytes have extremely large nuclei and thus the random movement of the kinases that diffuse in the region between cell membrane and the nucleus resembles a movement on a $2 \mathrm{D}$ manifold rather than in a $3 \mathrm{D}$ region.

In [23,24], guided by carefully prepared simulations, we have provided a rigorous proof that solutions to reaction diffusion equation for active kinase concentration $K$

$$
\frac{\partial K}{\partial t}=d \Delta K+F(K) \quad t \geq 0,
$$

in the thin shell between the nucleus and cell membrane, supplemented by Robin-type boundary condition on the outer membrane and by Neumann condition on the inner nuclear membrane:

$$
a R\left(1-K_{\text {out }}\right)=d n(\nabla K)_{\text {out }}, \quad n(\nabla K)_{\text {inn }}=0
$$

(where $a, d$ and $R$ are certain constants, and $F$ is a Lipschitz continuous function) are well approximated by the solutions of

$$
\frac{\partial K}{\partial t}=d \Delta_{\mathrm{LB}} K+F(K)+a R(1-K),
$$


where $\Delta_{\mathrm{LB}}$ is the Laplace-Beltrami operator on the unit sphere.

This result should but be treated as an instance of a general principle saying that in the thin layer approximation boundary conditions become integral parts of the limit master equation, as exemplified by the term $a R(1-K)$ 'jumping' from (1.4) to (1.5). This is precisely the perspective presented in [24]; see also computational examples presented there. The present paper, together with its companion [19], takes up the study and extends this principle to the case of transmission conditions.

\subsection{Comparison with the existing literature}

It should be noted here that, beginning with the seminal paper [46], considerable attention has already been given to thin layer approximation, both in the mathematical and in the physical literature; see, e.g., [7,9,26,34,74-77] for the former and [27] for an example of the latter. Our paper, however, differs from the previous works in several aspects.

First of all, we look at the problem of thin layer approximation from the perspective of convergence of semigroups of linear operators. Both the classical Trotter-KatoNeveu-like theorems and the more recently developed theory of 'degenerate' convergence to a semigroup that is defined only on a subspace of the original Banach space, have already proved to be instrumental in providing insight into phenomena originating in various fields of pure and applied mathematics. These include, but are not limited to, Markov processes [36], boundary theory for Markov chains [18], mathematical physics [72], and mathematical biology [17]; it is also perhaps worth noting the paper [62] where convergence of semigroups related to quite general boundary conditions is studied. Hence, our investigations may be seen as a reflection of our deep conviction that thin layer approximation, having intrinsic nature of a singular perturbation, could be seen as an integral part of the theory of degenerate convergence of semigroups of operators.

Secondly, in Sects. 3 and 4, we show that the theory of convergence of semigroups may be successfully applied to semigroups acting in the spaces of continuous functions. By contrast, the usual machinery used in the literature is the method of forms, and the analysis is usually carried out in the space of square integrable functions. This change of perspective is important for at least three reasons:

(i) Convergence of Feller semigroups is, as in the Trotter-Sova-Kurtz-Mackevičius Theorem (see, e.g., [51, p. 385]), equivalent to a weak convergence of the Markov processes involved, whereas a stochastic interpretation of a similar convergence result in a Hilbert space is rather unclear.

(ii) The analysis of the thin layer approximation hinges on a stretching transformation of 'thin coordinates' (see our Sect. 2.2, compare p. 111 in [74] or p. 583 in [68]). This transformation is an isometric isomorphism of appropriate spaces of continuous functions, but not of the related Hilbert spaces. 
(iii) Uniform convergence, i.e., convergence in the norm of the space of continuous functions, is stronger than that in the norm of $L^{2}$ (since the region we consider is bounded).

Notwithstanding the need for analysis in $C$, we must admit that, on the other hand, as exemplified also by the results of our Sect. 2, analytic tools available in $L^{2}$ are more flexible and thus allow treating potentially more general geometries and more general boundary conditions. Because of that our paper may be seen as an invitation for future analysis of the thin layer approximation in the context of stochastic processes. Perhaps stochastic analysis, and the results presented in $[28,58]$ in particular, may lead to generalizations that are also meaningful in this field.

The most significant difference between this paper and the existing literature is that, as heralded above, whereas our theorem is focused on the intriguing fact that boundary and transmission conditions in the limit become integral parts of the master equation, the previous papers are generally devoted to Neumann boundary conditions, which do not lead to such a phenomenon. An exception to this rule is the recent paper [68] which involves Robin-type boundary conditions; again, the analysis in that paper is carried out in Sobolev-type Hilbert space and allows treating more general geometries and boundary conditions than in our present paper, but the question of the role of boundary conditions in the limit and in the approximating equations is not discussed there. (Moreover, [68] is devoted to a completely different equation.) There are also remarks on Robin-type boundary conditions in [46,77], but they are of marginal character.

To the best of our knowledge, the fact that in the thin layer approximation boundary conditions 'jump into' the limit equation has first been discussed in [23,24]. Our analysis presented here and in the companion paper [19] extends this principle to transmission conditions also. It is our conviction that in the form involving transmission conditions the result gives better insight into the problem at hand. Moreover, it is more symmetrical and therefore more appealing aesthetically.

Lastly, the previous investigations were inspired mainly by physics, whereas our study, as already explained, was motivated by biology.

\subsection{Transmission conditions}

Equation (1.2) would not approximate (1.1) well, were transmission conditions supplementing (1.1) not chosen carefully; see Eqs. (2.3) and (3.1).

The first of these conditions may be plausibly interpreted by Newton's Law of Cooling, which says that the temperature at the membrane changes at a rate proportional to the difference of temperatures on its either sides, see [29, p. 9]. In this context, J. Crank uses the term radiation boundary condition. In a model of passing or diffusing through membranes, analogous transmission conditions were introduced by Tanner [82, Eq. (7)], who studied diffusion of particles through a sequence of permeable barriers (see also Powles et al. [73, Eq. (1.4)], for a continuation of the subject). In [2] (see, e.g., Eq. (4) there), similar conditions are used in describing absorption and desorption 
phenomena. We refer also to [40], where a compartment model with permeable walls (representing, e.g., cells, and axons in the white matter of the brain in particular) is analyzed, and to equation [42] there. Moreover, we acknowledge references [8,41,71], which we owe to an anonymous Referee, devoted to related models.

Conditions of type (2.3) were (re)-invented in [25] for the purpose of modeling so-called neurotransmitters and, drawing heavily on the fundamental work of Feller and Lévy on boundary behavior of stochastic processes (see, e.g., [50,52]), interpreted in probabilistic terms (see Chapters 3 and 11 in [17] for more details and bibliography on the subject, and [59] for a thorough stochastic analysis). An intuitive, if simplified, description of the so-called snapping out Brownian motion (the name coined in [59]), that is, a stochastic process related to (2.3) is as follows. A particle moves around chaotically on one side of the membrane and, as in the reflected Brownian motion, is being reflected from the membrane many times. The time 'spent at the membrane' is, however, measured and the larger is this time (and the larger is $\alpha$ or $\beta$, depending on which side of the membrane the particle is), the larger is the probability that the particle permeates through the membrane to its other side.

Conditions (3.1) describe yet more general rules: here, the membrane is sticky ( $p$ and $q$ are measures of stickiness of the membrane on its either sides), and thus, a particle diffusing on one side of the membrane may not only be reflected but also stay at the membrane for some time before it 'peels of' (see Chapter 3 in [17] or Example 3.59 in [60]). Additionally, when the particle permeates through the membrane, it randomly chooses its initial position for chaotic movement on the other side, according to the measure $\mu$ or $v$; in the process related to (2.3) this initial point is the point opposite to the point where the particle permeates.

Coming back to conditions of type (2.3), it is worth noting that they were extensively used in the papers $[15,21]$, devoted to the so-called averaging principle. This principle says that fast diffusions on a graph with edges separated by semi-permeable membranes situated on its vertices, or in the higher-dimensional regions separated by such membranes, are well approximated by a Markov chain with state-space constructed by identifying each edge with a point, or lumping all points of a region into one point. Also, remarkably, intensities of jumps in the limit process are functionals of permeability coefficients characterizing the approximating processes.

The similarity between the principle just described and the main result of the present paper is not accidental: whereas in [15,21] diffusion is fast in all directions, the thin layer approximation, as was already noted, is equivalent to an analysis of diffusion that is fast only in one direction.

\subsection{Nonlinearity}

Reaction-diffusion equations involve reaction terms, which in most important cases are nonlinear. Notably, it is these nonlinear terms that are responsible for many characteristic phenomena which cannot occur in a linear equation, such as system's bistability or existence of homo- and hetero-clinical waves. Since this research is motivated by 
problems in the theory of signaling pathways, and the phenomena mentioned above are critical for signal transmitting in living cells, our main result is stated for semilinear equations, that is for reaction-diffusion equations.

On the other hand, nonlinear terms in the reaction-diffusion equations employed in the theory of signaling pathways and, more generally, in mathematical biology often have strong continuity properties. For example, even if such a nonlinearity is not globally Lipschitz continuous from the outset, it is—as often as not—at least locally Lipschitz continuous. If this is the case and solutions of the equation can be proved (by checking Müller's conditions) to never leave certain regions of interest, analysis of such reaction-diffusion equations can be reduced to the analysis of equations with globally Lipschitz nonlinearity (see, e.g., Chapter 36 in [17]; see also Sect. 4 and the proof of Proposition 8.2 in [24]). Furthermore, by the main result of [22], solutions to semilinear problems with a globally Lipchitz nonlinear term converge (even in a degenerate manner) iff so do the solutions to the corresponding linear problems.

From the perspective of the latter result, it may seem that including nonlinear terms does not add value to the paper. Nevertheless, because of the motivations outlined above, we feel that disposing of these terms would make the results less natural and interesting (and the paper [22] is not so well known).

\subsection{Structure of the paper}

In Sect. 2, we state and prove our main theorem in the $L^{2}$ setting. In Sect. 3, we state this theorem in the context of $C$, and in Sect. 4, we present its proof.

\section{Analysis in $L^{2}$}

2.1. Intuitions and the limit master equation

Let $\mathcal{B}$ (' $\mathrm{b}$ ' for 'base') be a bounded, open subset of $\mathbb{R}^{2}$ with Lipschitz boundary. Given $\varepsilon \in(0,1]$, we consider the following 'split' solid (cylinder) $\Omega_{\varepsilon}=\Omega_{\varepsilon}^{-} \cup \Omega_{\varepsilon}^{+}$ formed by two bordering thin layers

$$
\begin{aligned}
& \Omega_{\varepsilon}^{-}:=\left\{(x, y, z) \in \mathbb{R}^{3}:(x, y) \in \mathcal{B},-\varepsilon<z<0\right\}, \\
& \Omega_{\varepsilon}^{+}:=\left\{(x, y, z) \in \mathbb{R}^{3}:(x, y) \in \mathcal{B}, 0<z<\varepsilon\right\} .
\end{aligned}
$$

This solid's lower and upper bases will be denoted

$$
\begin{aligned}
\mathcal{B}_{\varepsilon}^{\text {lo }}: & =\left\{(x, y, z) \in \mathbb{R}^{3}:(x, y) \in \mathcal{B}, z=-\varepsilon\right\}, \\
\mathcal{B}_{\varepsilon}^{\text {up }} & :=\left\{(x, y, z) \in \mathbb{R}^{3}:(x, y) \in \mathcal{B}, z=\varepsilon\right\} .
\end{aligned}
$$

We imagine that the plane separating the layers $\Omega_{\varepsilon}^{-}$and $\Omega_{\varepsilon}^{+}$is a semi-permeable membrane, and thus distinguish between what is 'right above' and 'right below' this 


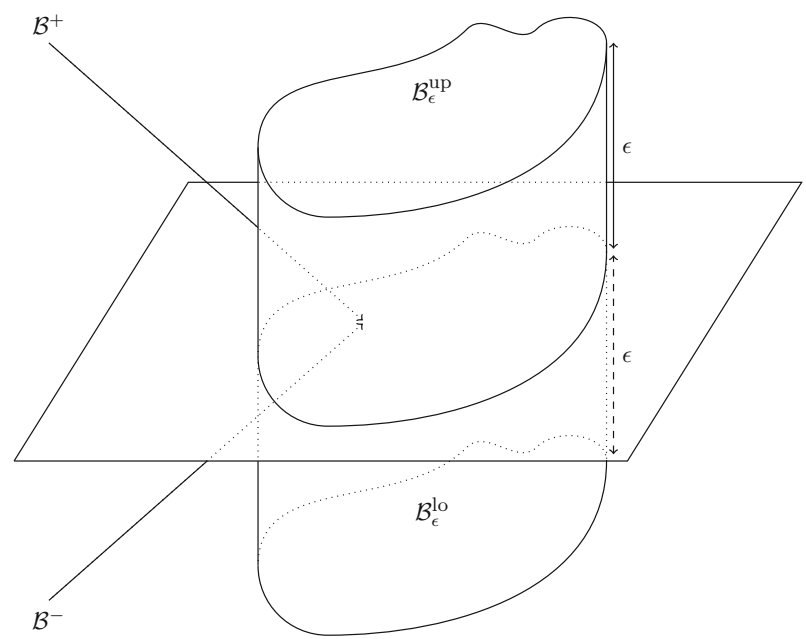

Figure 1. Two thin layers separated by a semi-permeable flat membrane

plane. Therefore, we introduce (see Fig. 1):

$$
\begin{aligned}
& \mathcal{B}^{-}:=\left\{(x, y, z) \in \mathbb{R}^{3}:(x, y) \in B, z=0-\right\} \\
& \mathcal{B}^{+}:=\left\{(x, y, z) \in \mathbb{R}^{3}:(x, y) \in B, z=0+\right\}
\end{aligned}
$$

Having prepared the stage for the analysis, we consider the following reactiondiffusion equation in $\Omega_{\varepsilon}$ :

$$
\partial_{t} u(t, x, y, z)=\Delta_{3 D} u(t, x, y, z)+F(u(t, x, y, z)),(x, y, z) \in \Omega_{\varepsilon}, t>0,
$$

where $\Delta_{3 D}=\partial_{x}^{2}+\partial_{y}^{2}+\partial_{z}^{2}$, and $F: \mathbb{R} \rightarrow \mathbb{R}$ in the reaction term is assumed globally Lipschitz continuous. In addition to usual Neumann boundary conditions on the sides of the solid, on the bases $\mathcal{B}_{\varepsilon}^{\text {up }}$ and $\mathcal{B}_{\varepsilon}^{\text {lo }}$ we impose Robin boundary conditions of the form

$$
\begin{aligned}
\partial_{z} u(t, x, y,-\varepsilon) & =\varepsilon c^{-}(x, y) u(t, x, y,-\varepsilon), \\
\partial_{z} u(t, x, y, \varepsilon) & =-\varepsilon c^{+}(x, y) u(t, x, y, \varepsilon), \quad(x, y) \in \mathcal{B}, t>0,
\end{aligned}
$$

where $c^{-}, c^{+}: \mathbb{R}^{2} \rightarrow \mathbb{R}$ are given, measurable, essentially bounded functions. As explained in $[23,24]$, the scaling factor (i.e., $\varepsilon$ ) is needed in these boundary conditions; otherwise the limit (as $\varepsilon \rightarrow 0$ ) discussed below will be uninteresting. These boundary conditions describe a stochastic mechanism of removing some of the diffusing particles touching the boundaries in regions where $c^{-}$and $c^{+}$are positive, and a similar mechanism of an inflow of new particles from regions of the boundary where $c^{-}$and $c^{+}$are negative. 
Moreover, on $\mathcal{B}^{-}$and $\mathcal{B}^{+}$we impose the following transmission conditions modeling semi-permeability of the membrane:

$$
\begin{aligned}
& \partial_{z} u(t, x, y, 0-)=\varepsilon \alpha(x, y)[u(x, y, 0-)-u(x, y, 0+)], \\
& \partial_{z} u(t, x, y, 0+)=\varepsilon \beta(x, y)[u(x, y, 0+)-u(x, y, 0-)], \quad(x, y) \in \mathcal{B}, t>0,
\end{aligned}
$$

where $\alpha, \beta: \mathbb{R}^{2} \rightarrow[0, \infty)$ are given, essentially bounded functions. These conditions describe a stochastic mechanism in which a particle in the upper layer may filter through the membrane to the lower layer, and vice versa. The functions $\alpha$ and $\beta$ are permeability coefficients: the larger is $\alpha$ in a given subset of the membrane, the shorter is the average time needed for a particle to filter through this part of the membrane from the lower to the upper layer, and the larger is the $\beta$ the shorter is the average time for a particle to filter from the upper to the lower layer (see [21,25,59], see also [17] p. 66 and the references given there). For our subsequent analysis (i.e., our generation and convergence results), the assumption that $\alpha$ and $\beta$ are non-negative is not needed; but a probabilistic interpretation of transmission conditions (2.3) with negative $\alpha$ and $\beta$ is less pleasing.

Our main objective is to study behavior of solutions to Eqs. (2.1)-(2.3) as $\varepsilon$ converges to 0 . We will argue that in this process, these solutions become more and more 'flat in the $z$-direction,' i.e., become less and less dependent on $z$, and thus in the limit may be regarded as functions of two variables (plus time). The functions obtained in the lower and upper layers differ, however, and thus will be denoted $u^{-}(t, x, y)$ and $u^{+}(t, x, y)$, respectively, and interpreted as functions on the upper and lower sides of the membrane. As it transpires, dynamics of $u^{-}$and $u^{+}$in time is governed by the master equation (1.2), where $F\left(u^{-}\right)$is a shorthand for the function $(x, y) \mapsto F\left(u^{-}(x, y)\right)$, and similarly with $F\left(u^{+}\right)$. This is a system of reaction-diffusion equations involving two-dimensional diffusion on the two sides of the membrane $\mathcal{B}$. As in [22-24], in the limit the boundary conditions (2.2) 'jump into' the main equation to form new terms of the reaction part. If $c^{-}$and $c^{+}$are non-negative, the terms

$$
-c^{-} u^{-} \text {and }-c^{+} u^{+}
$$

describe the process of random 'killing' of diffusing particles on the upper and lower sides, respectively. On the parts where $c^{-}$and $c^{+}$are negative, these terms model inflow of new diffusing particles. However, it is the matrix

$$
\left(\begin{array}{cc}
-\alpha & \alpha \\
\beta & -\beta
\end{array}\right)
$$

featuring in (1.2), that constitutes the most intriguing part of the limit system. As already explained, $\alpha$ and $\beta$ in (2.3) should be interpreted as permeability coefficients of the membrane. Here, they play a similar role: they should be interpreted as jump intensities: particles diffusing on, say, the lower side of the membrane, may jump to 
the upper side; in regions where $\alpha$ is large, expected times to such jumps are shorter than those in the regions where $\alpha$ is small. Similarly, a particle diffusing on the region of the upper side where $\beta$ is large will jump to the lower side on average quicker than a similar particle diffusing in a region where $\beta$ is small. Remarkably, if $\alpha=\beta=0$, (2.3) reduces to Neumann boundary conditions: the membrane is non-permeable, and diffusing particles in lower and upper layers never filter to the other side. A similar observation may be made concerning (1.2): for $\alpha=\beta=0$, the system is uncoupled: the are no jumps between the lower and upper sides.

\subsection{A view through a magnifying glass}

To see that solutions to (2.1)-(2.3) gradually lose dependence on $z$ variable, we look at $\Omega_{\varepsilon}$ through a magnifying glass, by introducing the change of variables, $\tilde{z}=\varepsilon^{-1} z$, which transforms $\Omega_{\varepsilon}$ into

$$
\Omega:=\Omega_{1}
$$

To this end, we write $\tilde{u}(t, x, y, \tilde{z})=u(t, x, y, \epsilon \tilde{z})$. Then, equation (2.1) transforms to

$$
\partial_{t} \tilde{u}(t, x, y, \tilde{z})=\left(\partial_{x}^{2}+\partial_{y}^{2}+\varepsilon^{-2} \partial_{z}^{2}\right) \tilde{u}(t, x, y, \tilde{z})+F(\tilde{u}(t, x, y, \tilde{z})),
$$

for $(x, y, \tilde{z}) \in \Omega, t>0$ while the boundary conditions (2.2) become

$$
\begin{aligned}
\partial_{\tilde{z}} \tilde{u}(t, x, y,-1) & =\varepsilon^{2} c^{-}(x, y) \tilde{u}(x, y,-1), \\
\partial_{\tilde{z}} \tilde{u}(t, x, y, 1) & =-\varepsilon^{2} c^{+}(x, y) \tilde{u}(x, y, 1), \quad x, y \in \mathbb{R}, t>0 .
\end{aligned}
$$

Similarly, the transmission conditions become

$$
\begin{aligned}
& \partial_{\tilde{z}} \tilde{u}(t, x, y, 0-)=\varepsilon^{2} \alpha(x, y)[\tilde{u}(x, y, 0-)-\tilde{u}(x, y, 0+)], \\
& \partial_{\tilde{z}} \tilde{u}(t, x, y, 0+)=\varepsilon^{2} \beta(x, y)[\tilde{u}(x, y, 0+)-\tilde{u}(x, y, 0-)], \quad x, y \in \mathbb{R}, t>0 .
\end{aligned}
$$

\subsection{A generation theorem in $L^{2}(\Omega)$}

For notational simplicity we drop the tildes, and then rewrite system (2.5)-(2.7) as an abstract evolution equation on the space $L^{2}(\Omega)$, as follows:

$$
\partial_{t} u_{\varepsilon}(t)=A_{\varepsilon} u_{\varepsilon}(t)+F\left(u_{\varepsilon}(t)\right), \quad u_{\varepsilon}(0)=\stackrel{0}{u} \in L^{2}(\Omega)
$$

where $u_{\varepsilon}:[0, \infty) \rightarrow L^{2}(\Omega)$ and $A_{\varepsilon}$ is a suitable realization of the differential operator $\partial_{x}^{2}+\partial_{y}^{2}+\varepsilon^{-2} \partial_{z}^{2}$, subject to the boundary and transmission conditions (2.6)-(2.7) (see later on). The reaction term $F$, although denoted by the same letter as the function featuring in (2.1), has a slightly different meaning. Namely, for a $u \in L^{2}(\Omega)$ we may define

$$
(\mathrm{F}(u))(x, y, z):=F(u(x, y, z))
$$


where $F$ on the right-hand side is the function from (2.1). Assuming that $F(0)=0$ or, more generally, that there is a $u \in L^{2}(\Omega)$ such that $\mathrm{F}(u) \in L^{2}(\Omega)$, we check, using the existence of a global Lipschitz constant for $F$, that (2.9) defines a globally Lipschitz continuous map $L^{2}(\Omega) \rightarrow L^{2}(\Omega)$, with the Lipschitz constant inherited from $F$. In (2.8), for simplicity of notation, we do not distinguish between $F$ and $\mathrm{F}$.

As discussed in [22] and our Sect. 1.5, the problems of well-posedness and convergence of solutions to (2.8), reduce to these for the related problem without the nonlinear term (see also the end of Sect. 2.4):

$$
\partial_{t} u_{\varepsilon}(t)=A_{\varepsilon} u_{\varepsilon}(t), \quad u_{\varepsilon}(0)=\stackrel{o}{u} \in L^{2}(\Omega) .
$$

We start by establishing well-posedness of the problem (2.10) making use of the theory of sesquilinear forms (see, e.g., [53,67] for details of this theory).

We recall that if $H$ is a complex Hilbert space, a sesquilinearform on $H$ is a mapping $\mathfrak{a}: D(\mathfrak{a}) \times D(\mathfrak{a}) \rightarrow \mathbb{C}$ which is linear in the first component and antilinear in the second component. A form $\mathfrak{a}$ is called accretive if $\Re \mathfrak{a}[u, u] \geq 0$ for all $u \in D(\mathfrak{a})$; it is called closed, if $D(\mathfrak{a})$ is a Hilbert space with respect to the inner product $[u, v]_{\mathfrak{a}}=$ $\Re \mathfrak{a}[u, v]+[u, v]_{H}$. A sesquilinear form is called densely defined, if $D(\mathfrak{a})$ is dense in $H$. It is called symmetric, if $\mathfrak{a}[u, v]=\mathfrak{a}[v, u]$. As customary, we will write $\mathfrak{a}[u]$ for $\mathfrak{a}[u, u]$.

Given an accretive and closed sesquilinear form $\mathfrak{a}$ that is densely defined, we can define the associated operator $A$ by setting

$$
D(A)=\left\{u \in D(\mathfrak{a}): \exists f \in H: \mathfrak{a}[u, v]=-[f, v]_{H} \forall v \in D(\mathfrak{a})\right\}
$$

and $A u:=f$. We thus have $\mathfrak{a}[u, v]=-[A u, v]_{H}$ for all $u \in D(A)$ and $v \in D(\mathfrak{a})$. It is well known that the operator $A$ associated with an accretive, densely defined and closed sesquilinear form, is the generator of an analytic contraction semigroup on the space $H$.

Our goal in this section is to find, for each $\varepsilon \in(0,1]$, a sesquilinear form $\mathfrak{a}_{\varepsilon}$ such that

$$
A_{\varepsilon}:=\partial_{x}^{2}+\partial_{y}^{2}+\varepsilon^{-2} \partial_{z}^{2}
$$

with boundary and transmission conditions (2.6)-(2.7) is its associated operator. To this end, we define $\mathfrak{H} \subset L^{2}(\Omega)$ by

$$
\mathfrak{H}:=\left\{v \in L^{2}(\Omega): v_{\mid \Omega^{+}} \in H^{1}\left(\Omega^{+}\right), v_{\mid \Omega^{-}} \in H^{1}\left(\Omega^{-}\right)\right\},
$$

where $\Omega^{+}:=\Omega_{1}^{+}$and $\Omega^{-}:=\Omega_{1}^{-}$; this is going to be the domain of the forms $\mathfrak{a}_{\varepsilon}, \varepsilon \in(0,1]$. We recall (see, e.g., [1] Part I, Case C of Theorem 4.12) that each $w \in H^{1}\left(\Omega^{+}\right)$leaves square integrable traces on $\mathcal{B}^{\text {up }}:=\mathcal{B}_{1}^{\text {up }}$ and $\mathcal{B}^{+}$(denoted $w(x, y, 1)$ and $w(x, y, 0+)$, respectively), and that the trace operators are continuous. Similarly, each $w \in H^{1}\left(\Omega^{-}\right)$leaves square integrable traces on $\mathcal{B}^{\text {lo }}:=\mathcal{B}_{1}^{\text {lo }}$ and $\mathcal{B}^{-}$(denoted $w(x, y,-1)$ and $w(x, y, 0-)$, respectively), and the trace operators are 
bounded. Hence, a $v \in \mathfrak{H}$ leaves square integrable traces on each of these four sets, and we have the following four bounded trace operators:

$$
\begin{gathered}
T^{\text {up }: \mathfrak{H}} \rightarrow L^{2}\left(\mathcal{B}^{\text {up }}\right), T^{\text {lo }}: \mathfrak{H} \rightarrow L^{2}\left(\mathcal{B}^{\text {lo }}\right), \\
T^{+}: \mathfrak{H} \rightarrow L^{2}\left(\mathcal{B}^{+}\right), T^{-}: \mathfrak{H} \rightarrow L^{2}\left(\mathcal{B}^{-}\right),
\end{gathered}
$$

where $\mathfrak{H}$ is equipped with the norm

$$
\|v\|_{\mathfrak{H}}=\sqrt{\left\|v_{\mid \Omega^{+}}\right\|_{H^{1}\left(\Omega^{+}\right)}^{2}+\left\|v_{\mid \Omega^{-}}\right\|_{H^{1}\left(\Omega^{-}\right)}^{2}} .
$$

Finally, let

$$
D_{\varepsilon} \subset L^{2}(\Omega)
$$

be composed of $u$ such that $v_{\mid \Omega^{+}} \in H^{2}\left(\Omega^{+}\right), v_{\mid \Omega^{-}} \in H^{2}\left(\Omega^{-}\right)$and such that boundary and transmission conditions (2.6)-(2.7) are satisfied in the weak sense (see, e.g., [21] Sect. 4.2 for details). For such $u$, we define $A_{\varepsilon} u$ as $\left(\partial_{x}^{2}+\partial_{y}^{2}+\varepsilon^{-2} \partial_{z}^{2}\right) u$.

With these definitions, we take $u \in D_{\varepsilon}$ and $v \in \mathfrak{H}$, multiply $A_{\varepsilon} u$ by $\bar{v}$, and integrate the product over $\Omega^{+}$. Integration by parts formula then shows that

$$
\begin{aligned}
\int_{\Omega^{+}} & \left(A_{\varepsilon} u\right) \bar{v}(x, y, z) \mathrm{d}(x, y, z) \\
= & -\int_{\Omega^{+}}\left[\partial_{x} u \partial_{x} \bar{v}+\partial_{y} u \partial_{y} \bar{v}+\varepsilon^{-2} \partial_{z} u \partial_{z} \bar{v}\right](x, y, z) \mathrm{d}(x, y, z) \\
& +\varepsilon^{-2} \int_{\mathcal{B}} \partial_{z} u(x, y, 1) \bar{v}(x, y, 1) \mathrm{d}(x, y) \\
& -\varepsilon^{-2} \int_{\mathcal{B}} \partial_{z} u(x, y, 0+) \bar{v}(x, y, 0+) \mathrm{d}(x, y) \\
= & -\int_{\Omega^{+}}\left[\partial_{x} u \partial_{x} \bar{v}+\partial_{y} u \partial_{y} \bar{v}+\varepsilon^{-2} \partial_{z} u \partial_{z} \bar{v}\right] \mathrm{d}(x, y, z) \\
& -\int_{\mathcal{B}} c^{+}(x, y) u(x, y, 1) \bar{v}(x, y, 1) \mathrm{d}(x, y) \\
& -\int_{\mathcal{B}} \beta(x, y)[u(x, y, 0+)-u(x, y, 0-)] \bar{v}(x, y, 0+) \mathrm{d}(x, y) .
\end{aligned}
$$

Here, in the second step, we used the first of the boundary conditions (2.6) and the first of the transmission conditions (2.7). Similarly, we check that

$$
\begin{aligned}
\int_{\Omega^{-}} & \left(A_{\varepsilon} u\right) \bar{v}(x, y, z) \mathrm{d}(x, y, z) \\
= & -\int_{\Omega^{-}}\left[\partial_{x} u \partial_{x} \bar{v}+\partial_{y} u \partial_{y} \bar{v}+\varepsilon^{-2} \partial_{z} u \partial_{z} \bar{v}\right](x, y, z) \mathrm{d}(x, y, z) \\
& -\int_{\mathcal{B}} c^{-}(x, y) u(x, y,-1) \bar{v}(x, y,-1) \mathrm{d}(x, y) \\
& -\int_{\mathcal{B}} \alpha(x, y)[u(x, y, 0-)-u(x, y, 0+)] \bar{v}(x, y, 0-) \mathrm{d}(x, y) .
\end{aligned}
$$


This calculation suggests that, for $u, v \in \mathfrak{H}$, we should define

$$
\begin{aligned}
\mathfrak{a}_{\varepsilon}[u, v]:= & \int_{\Omega}\left[\partial_{x} u \partial_{x} \bar{v}+\partial_{y} u \partial_{y} \bar{v}+\varepsilon^{-2} \partial_{z} u \partial_{z} \bar{v}\right](x, y, z) \mathrm{d}(x, y, z) \\
& +\int_{\mathcal{B}} c^{+}(x, y) u(x, y, 1) \bar{v}(x, y, 1) \mathrm{d}(x, y) \\
& +\int_{\mathcal{B}} c^{-}(x, y) u(x, y,-1) \bar{v}(x, y,-1) \mathrm{d}(x, y) \\
& +\int_{\mathcal{B}} \beta(x, y)[u(x, y, 0+)-u(x, y, 0-)] \bar{v}(x, y, 0+) \mathrm{d}(x, y) \\
& +\int_{\mathcal{B}} \alpha(x, y)[u(x, y, 0-)-u(x, y, 0+)] \bar{v}(x, y, 0-) \mathrm{d}(x, y) .
\end{aligned}
$$

The following proposition is a particular case of Proposition 5.1 in [21]. For the sake of completeness, we present its proof in Appendix.

Proposition 1. Forms $\mathfrak{a}_{\varepsilon}$ are densely defined and closed. Furthermore, there is a $\gamma>0$ such that for all $\varepsilon \in(0,1]$,

$$
\left|\mathfrak{s}\left(\mathfrak{a}_{\varepsilon}+\gamma\right)[u]\right| \leq \Re\left(\mathfrak{a}_{\varepsilon}+\gamma\right)[u], \quad u \in \mathfrak{H} .
$$

Inequality (2.16) shows in particular that forms $\mathfrak{a}_{\varepsilon}+\gamma$ are accretive. Thus, the related operators are generators of holomorphic contraction semigroups. These operators are equal to $A_{\varepsilon}-\gamma I$ where $I$ is the identity operator in $L^{2}(\Omega)$, and $\left(A_{\varepsilon}, D\left(A_{\varepsilon}\right)\right)$ is the operator related to the form $\mathfrak{a}_{\varepsilon}$. Calculations (2.13) and (2.14) show that $\left(A_{\varepsilon}, D\left(A_{\varepsilon}\right)\right)$ is an extension of $\left(A_{\varepsilon}, D_{\varepsilon}\right)$. We may thus write

$$
\left\|\mathrm{e}^{t A_{\varepsilon}}\right\| \leq \mathrm{e}^{\gamma t}
$$

here, and in what follows, $A_{\varepsilon}$ is always considered with domain $D\left(A_{\varepsilon}\right)$. However, inequality (2.16), reveals much more: the forms $\mathfrak{a}_{\varepsilon}+\gamma$ are uniformly sectorial and so are the semigroups generated by $A_{\varepsilon}-\gamma I$. This information will be of crucial importance in the next section.

\subsection{Convergence as $\varepsilon \rightarrow 0$}

Finally, we want to let $\varepsilon \rightarrow 0$. To that end, we use a convergence theorem for uniformly sectorial forms due to Ouhabaz [66] which generalizes the convergence theorem of Simon [80], who dealt with the case of symmetric forms. To explain: the forms

$$
\tilde{\mathfrak{a}}_{\varepsilon}:=\mathfrak{a}_{\varepsilon}+\gamma,
$$

in addition to being accretive and uniformly sectorial, have the following properties:

(a) they have the same domain (i.e., $\mathfrak{H}$ ) and $\mathfrak{R} \tilde{\mathfrak{a}}_{\varepsilon}[u] \leq \mathfrak{R} \tilde{\mathfrak{a}}_{\mathcal{E}^{\prime}}[u]$ for all $u \in \mathfrak{H}$, provided $\varepsilon \geq \varepsilon^{\prime}$ (which is to say that $\Re \widetilde{\mathfrak{a}}_{\varepsilon}[u]$ increases as $\varepsilon$ decreases), 
(b) the imaginary parts of $\tilde{\mathfrak{a}}_{\varepsilon}[u]$ do not depend on $\varepsilon$.

Ouhabaz shows that in such a case (and in a more general situation), the form $\widetilde{\mathfrak{b}}[u]:=$ $\lim _{\varepsilon \rightarrow 0} \widetilde{\mathfrak{a}}_{\varepsilon}[u]$ (extended via polarization formula), defined on the domain

$$
D(\widetilde{\mathfrak{b}})=\left\{u \in \mathfrak{H} ; \sup _{\varepsilon \in(0,1]} \widetilde{\mathfrak{a}}_{\varepsilon}[u]<\infty\right\}
$$

is accretive, closed and sectorial (so that (2.16) holds for all $u \in D(\widetilde{\mathfrak{b}})$, if $\mathfrak{a}_{\varepsilon}$ is replaced by $\mathfrak{b}:=\widetilde{\mathfrak{b}}-\gamma)$. As we shall see soon, in our case, in distinction to the situation considered by Ouhabaz, this form is not densely defined. Hence, the related operator, say $B+\gamma I$ (where $B$ is the operator related to $\mathfrak{b}$ ), generates a holomorphic semigroup merely on the subspace $H_{0}:=\overline{D(\widetilde{b})}$. Nevertheless, Ouhabaz's arguments may be extended to this case to show that

$$
\lim _{\varepsilon \rightarrow 0}\left(\mu I-A_{\varepsilon}-\gamma I\right)^{-1}=(\mu I-B-\gamma I)^{-1} P,
$$

strongly, for all $\mu$ in a sector of the complex plane (see the comment on p. 676 in [21]). Here, $P$ is the orthogonal projection onto $H_{0}$. Using straightforward arguments involving contour integrals, presented in more detail in, e.g., [10] or [17] Chapter 31, one then deduces that $\lim _{\varepsilon \rightarrow 0} \mathrm{e}^{-\gamma t} \mathrm{e}^{t A_{\varepsilon}}=\mathrm{e}^{-\gamma t} \mathrm{e}^{t B} P$ (strongly). In other words, we have the following theorem.

Theorem 1. We have

$$
\lim _{\varepsilon \rightarrow 0} \mathrm{e}^{t A_{\varepsilon}}=\mathrm{e}^{t B} P, \quad t>0
$$

For $u \in H_{0}$ this limit is uniform for $t$ in compact subsets of $[0, \infty)$; for other $u$ it is uniform for $t$ in compact subsets of $(0, \infty)$.

Hence, we are left with the task of characterizing $D(\widetilde{\mathfrak{b}})=D(\mathfrak{b})$, the form $\mathfrak{b}$, and the related operator $B$. We want to check to see that the limit dynamics is governed by (1.2) (with $F=0$ ).

The only term in the definition of $\widetilde{\mathfrak{a}}_{\varepsilon}[u]$ (see (2.15)) that involves $\varepsilon$ is

$$
\varepsilon^{-2} \int_{\Omega}\left|\partial_{z} u\right|^{2}(x, y, z) \mathrm{d}(x, y, z) ;
$$

it is thus clear that $\sup _{\varepsilon \in(0,1]} \widetilde{\mathfrak{a}}_{\varepsilon}[u]<\infty$ implies that $\partial_{z} u=0$ almost everywhere, i.e., that $u$ does not depend on $z$. Conversely, if $u$ does not depend on $z$, then the supremum in question exists, because no term in the definition depends on $\varepsilon$. Thus, more specifically, for $u \in D(\widetilde{\mathfrak{b}})=D(\mathfrak{b})$ there are functions $u^{-}, u^{+} \in H^{1}(\mathcal{B})$ such that

$$
\begin{aligned}
& u(x, y, z)=u^{-}(x, y), \quad z \in(-1,0), \\
& u(x, y, z)=u^{+}(x, y), \quad z \in(0,1),
\end{aligned}
$$


almost surely in $(x, y, z)$. Hence, any $u$ may be identified with a pair of elements of $H^{1}(\mathcal{B})$ and $\overline{D(\mathfrak{b})}$ may be identified with the direct sum of two copies of $L^{2}(\mathcal{B})$. Moreover, by polarization formula, for $u$ and $v$ in $D(\mathfrak{b})$,

$$
\begin{aligned}
\mathfrak{b}[u, v]:= & \int_{\mathcal{B}}\left[\partial_{x} u^{+} \partial_{x} \bar{v}^{+}+\partial_{y} u^{+} \partial_{y} \bar{v}^{+}\right] \mathrm{d} \lambda_{2} \\
& +\int_{\mathcal{B}}\left[\partial_{x} u^{-} \partial_{x} \bar{v}^{-}+\partial_{y} u^{-} \partial_{y} \bar{v}^{-}\right] \mathrm{d} \lambda_{2} \\
& +\int_{\mathcal{B}} c^{+} u^{+} \bar{v}^{+} \mathrm{d} \lambda_{2}+\int_{\mathcal{B}} c^{-} u^{-} \bar{v}^{-} \mathrm{d} \lambda_{2} \\
& +\int_{\mathcal{B}} \alpha\left(u^{-}-u^{+}\right) \bar{v}^{-} \mathrm{d} \lambda_{2}+\int_{\mathcal{B}} \beta\left(u^{+}-u^{-}\right) \bar{v}^{+} \mathrm{d} \lambda_{2},
\end{aligned}
$$

where $\lambda_{2}$ is the two-dimensional Lebesgue measure. It is clear that the two terms in the third line here can be extended to the form defined on the entire $L^{2}(\mathcal{B}) \times L^{2}(\mathcal{B})$, and that the associated operator is bounded and given by:

$$
\left(\begin{array}{l}
u^{-} \\
u^{+}
\end{array}\right) \mapsto-\left(\begin{array}{l}
c^{-} u^{-} \\
c^{+} u^{+}
\end{array}\right) \text {. }
$$

We see the same operator also in (1.2). Similarly, the terms in the fourth line come from the bounded operator in $L^{2}(\mathcal{B}) \times L^{2}(\mathcal{B})$ which may be represented by the matrix (2.4). Moreover, the first term is well known: the associated operator is the Neumann Laplace operator $\Delta_{2 D}$ (i.e., the $2 D$ Laplace operator with Neumann boundary conditions) in $L^{2}(\mathcal{B})$ - see, e.g., Example 8.1.6 in [4], and an analogous remark concerns the second term. Thus, the first two terms are associated with the operator

$$
\left(\begin{array}{l}
u^{-} \\
u^{+}
\end{array}\right) \mapsto\left(\begin{array}{c}
\Delta_{2 D} u^{-} \\
\Delta_{2 D} u^{+}
\end{array}\right),
$$

and the operator related to the entire limit form may be represented as

$$
B=\left(\begin{array}{cc}
\Delta_{2 D}-c^{-} & 0 \\
0 & \Delta_{2 D}-c^{+}
\end{array}\right)+\left(\begin{array}{cc}
-\alpha & \alpha \\
\beta & -\beta
\end{array}\right) .
$$

In other words, formula (2.17) shows that mild solutions of (2.1)-(2.3) with initial condition $\stackrel{o}{u} \in L^{2}(\Omega)$, and nonlinear term equal 0 , converge to those of (1.2) with initial condition $P \stackrel{o}{u}=\left(P^{-} \stackrel{\circ}{u}, P^{+}{ }_{u}^{\circ}\right)$ where

$$
P^{-} \stackrel{\mathrm{o}}{u}(x, y)=\int_{-1}^{0} \stackrel{\mathrm{o}}{u}(x, y, z) \mathrm{d} z \quad P^{+} \stackrel{\mathrm{o}}{u}(x, y)=\int_{0}^{1} \stackrel{\mathrm{o}}{u}(x, y, z) \mathrm{d} z .
$$

Hence, it remains to take care of the nonlinearity. By the main theorem of [22], however, convergence of semigroups $\left(\mathrm{e}^{t A_{\varepsilon}}\right)_{t \geq 0}$, even in a degenerate manner, as in (2.17), implies convergence of mild solutions of (2.1)-(2.3) to solutions of

$$
\partial_{t} u(t)=B u(t)+P F(u(t)) .
$$

Moreover, since $F$ (or, in fact, F, see (2.9)) leaves $H_{0}$ invariant, $P$ is not needed on the right-hand side here. We have proved the following theorem. 
Theorem 2. Mild solutions of (2.1)-(2.3) with initial condition $\stackrel{o}{u} \in L^{2}(\Omega)$ converge to those of (1.2) with initial condition P $\stackrel{o}{u}$.

\section{Analysis in $C$ : the main result and robustness}

For an analogous result in the space of continuous functions, we need more restrictive assumptions on the base $\mathcal{B}$ : we assume that $\mathcal{B}$ is a bounded, connected and open set, and that its boundary $\partial \mathcal{B}$ is of class $C^{2, \kappa}, \kappa \in(0,1]$ (see [36] p. 368). This allows concluding that the Neumann Laplace operator with suitable domain in $C(\mathcal{B})$ is closable and its closure is a Feller generator in $C(\mathcal{B})$ ([36] p. 369). For technical reasons, we also need to be content with constant permeability coefficients $\alpha$ and $\beta$. On the other hand, we generalize the mechanism of filtering through the membrane. More specifically, given parameters $p, q \in[0,1]$ and Borel probability measures $\mu$ and $v$ on $[-1,0]$ and $[0,1]$, respectively, we consider the following transmission conditions describing permeability of the membrane separating the lower and upper layers:

$$
\begin{aligned}
& \left(\varepsilon p \partial_{z}^{2}+(1-p) \partial_{z}\right) u(t, x, y, 0-)=\varepsilon \alpha\left[\int_{0+}^{\varepsilon} u(t, x, y, z) v_{\varepsilon}(\mathrm{d} z)-u(t, x, y, 0-)\right] \\
& \left(\varepsilon q \partial_{z}^{2}-(1-q) \partial_{z}\right) u(t, x, y, 0+)=\varepsilon \beta\left[\int_{-\varepsilon}^{0-} u(t, x, y, z) \mu_{\varepsilon}(\mathrm{d} z)-u(t, x, y, 0+)\right]
\end{aligned}
$$

where $(x, y) \in \mathcal{B}, t>0, \mu_{\varepsilon}$ is the transport of $\mu$ to $[-\varepsilon, 0-]$ (via the map $x \mapsto-\varepsilon x$ ), and $v_{\varepsilon}$ is the transport of $v$ to $[0+, \varepsilon]$ via $x \mapsto \varepsilon x$. Again, epsilons in these transmission conditions are arranged in such a way that the limit as $\varepsilon \rightarrow 0$ is non-trivial. Remarkably, if $p=q=1$ the epsilons cancel out, i.e., no scaling is needed.

As already noted, in comparison to (2.3), these boundary conditions describe a more general way Brownian particles on one side of the membrane may filter to the other side (see also Sect. 4.2): the additional term with the second derivative speaks of the possibility for a particle to stick to the membrane for some random time. In particular, for $p=1$ the particles are stopped at the lower side of the membrane, and after an exponential time (with parameter $\alpha$ ) released to jump to the upper side. The measures $\mu_{\varepsilon}$ and $v_{\varepsilon}$ describe a random position of a particle after it filters from one side of the membrane to the other. For $p=q=0$ and $\mu=\delta_{0}, v=\delta_{0}$ where $\delta_{0}$ is the Dirac measure concentrated at 0 , transmission conditions (3.1) reduce to (2.3).

As in Sect. 2.2, instead of working with 'the same equation' but in varying spaces $C\left(\Omega_{\varepsilon}\right), \varepsilon \in(0,1]$ of continuous functions on thinner and thinner domains $\Omega_{\varepsilon}=$ $\Omega_{\varepsilon}^{-} \cup \Omega_{\varepsilon}^{+}$where, this time,

$$
\begin{aligned}
& \Omega_{\varepsilon}^{-}:=\left\{(x, y, z) \in \mathbb{R}^{3}:(x, y) \in \mathcal{B},-\varepsilon \leq z \leq 0-\right\}, \\
& \Omega_{\varepsilon}^{+}:=\left\{(x, y, z) \in \mathbb{R}^{3}:(x, y) \in \mathcal{B}, 0+\leq z \leq \varepsilon\right\},
\end{aligned}
$$


we blow up the thin coordinate $z$ by dividing it by $\varepsilon$, to work with a family of equations in a single reference space

$$
C(\Omega):=C\left(\Omega_{1}\right)
$$

Notably, this transformation is an isometric isomorphism of the spaces of continuous functions involved (but not of the $L^{2}$-type spaces considered in Sect. 2): we are using a magnifying glass and not a distorting mirror.

The family of equations in $C(\Omega)$ we obtain is of the form

$$
\partial_{t} u(t)=\overline{\mathfrak{A}_{\varepsilon}} u(t), \quad u(0)=\stackrel{o}{u} \in C(\Omega),
$$

where $\mathfrak{A}_{\varepsilon}=\partial_{x}^{2}+\partial_{y}^{2}+\varepsilon^{-2} \partial_{z}^{2}$ and $D\left(\mathfrak{A}_{\varepsilon}\right)$ is composed of $u \in C(\Omega)$ such that (a) when restricted to either of $\Omega^{-}$or $\Omega^{+}$they are of class $C^{2}$ and for each $z \in$ $[-1,0-] \cup[0+, 1],(x, y) \mapsto u(x, y, z)$ is of class $C^{2, \kappa}$ and (b) besides Neumann boundary conditions on the boundary of $\Omega$, they satisfy the following transmission conditions on the membrane separating the upper and lower parts of $\Omega$ :

$$
\begin{aligned}
\left(p \partial_{z}^{2}+(1-p) \partial_{z}\right) u(x, y, 0-) & =\varepsilon^{2} \alpha\left[v_{x, y}(u)-u(x, y, 0-)\right] \\
\left(q \partial_{z}^{2}-(1-q) \partial_{z}\right) u(x, y, 0+) & =\varepsilon^{2} \beta\left[\mu_{x, y}(u)-u(x, y, 0+)\right]
\end{aligned}
$$

where $(x, y) \in \mathcal{B}$, whereas $v_{x, y}(u)$ and $v_{x, y}(u)$ are shorthands for

$$
\int_{[0+, 1]} u(x, y, z) v(\mathrm{~d} z) \text { and } \int_{[-1,0-]} u(x, y, z) \mu(\mathrm{d} z),
$$

respectively. As we shall see later, $\mathfrak{A}_{\varepsilon}, \varepsilon \in(0,1]$ are closable and their closures $\overline{\mathfrak{A}_{\varepsilon}}, \varepsilon \in(0,1]$ are conservative Feller generators (see Proposition 4). Our goal is to study the $\operatorname{limit}_{\lim _{\varepsilon \rightarrow 0}} \mathrm{e}^{t \overline{\mathfrak{A}_{\varepsilon}}}$.

We note the absence of the nonlinear term in (3.2). As discussed above, a convergence result for semigroups implies also convergence of solutions of the related semi-linear equations (with globally Lipschitz continuous nonlinearity), and thus, we disposed of this term without loss of generality. Furthermore, we note that, for simplicity of exposition, since the role of $c^{-}$and $c^{+}$of Sect. 2 has been already explained in [22-24], and we want to focus on the more intriguing role of $\alpha$ and $\beta$, it is assumed that $c^{-}$and $c^{+}$are now zero (since Neumann boundary conditions on the entire boundary of $\Omega$ are assumed).

As a preparation for the main theorem in this section, let $C^{b}(\Omega) \subset C(\Omega)$ be the subspace of $u \in C(\Omega)$ that do not depend on $z$ (i.e., are 'flat' in the $z$ direction). For each member $u$ of $C^{b}(\Omega)$ there are two continuous functions, say $u^{-}$and $u^{+}$, on $\mathcal{B}$ such that

$$
\begin{array}{ll}
u(x, y, z)=u^{-}(x, y), & (x, y) \in \mathcal{B}, z \in[-1,0-], \\
u(x, y, z)=u^{+}(x, y), & (x, y) \in \mathcal{B}, z \in[0+, 1],
\end{array}
$$


and thus, $u$ may be identified with $\left(u^{-}, u^{+}\right) \in C(\mathcal{B}) \times C(\mathcal{B})$. In other words, $C^{b}(\Omega)$ is isometrically isomorphic to the latter Cartesian product. By the generation theorem from p. 369 in [36], the Neumann Laplace operator, say $\Delta_{2 D}$, is closable and its closure $\overline{\Delta_{2 D}}$ generates a Feller semigroup in $C(\mathcal{B})$. Thus, by the Phillips perturbation theorem, the operator

$$
\mathfrak{B}=\left(\begin{array}{cc}
\overline{\Delta_{2 D}} & 0 \\
0 & \Delta_{2 D}
\end{array}\right)+\left(\begin{array}{cc}
-\alpha & \alpha \\
\beta & -\beta
\end{array}\right),
$$

with domain $D\left(\overline{\Delta_{2 D}}\right) \times D\left(\overline{\Delta_{2 D}}\right)$ is a generator in $C(\mathcal{B}) \times C(\mathcal{B})$. (Also, if the latter space is appropriately identified with the space of continuous functions on two copies of $\mathcal{B}$, the operator $\mathfrak{B}$ may be seen to be a conservative Feller generator.) Our main theorem says that this $\mathfrak{B}$ governs the limit evolution.

Theorem 3. We have,

$$
\lim _{\varepsilon \rightarrow 0} \mathrm{e}^{t \overline{\mathfrak{A}_{\varepsilon}}} u=\mathrm{e}^{t \mathfrak{B}} \mathcal{P}_{p, q} u, \quad u \in C(\Omega), t>0
$$

where $\mathcal{P}_{p, q}$ is the projection on $C^{b}(\Omega)$ defined by

$$
\mathcal{P}_{p, q} u=\left(u_{1}, u_{2}\right)
$$

with

$$
\begin{aligned}
& u_{1}(x, y)=p u(x, y, 0-)+(1-p) \int_{-1}^{0} u(x, y, z) \mathrm{d} z, \\
& u_{2}(x, y)=q u(x, y, 0+)+(1-q) \int_{0}^{1} u(x, y, z) \mathrm{d} z, \quad(x, y) \in \mathcal{B} .
\end{aligned}
$$

The intuition behind this theorem is as follows. As $\varepsilon \rightarrow 0$, diffusion in the vertical direction becomes faster and faster, and the solutions to (3.2) become more and more flat in this direction. Therefore, in the limit they resemble functions of two variables, defined on the two sides of the separating membrane. On each of these sides, we have diffusion (with reflection on the boundary), and these sides communicate via jumps, as expressed in the second matrix defining the operator $\mathfrak{B}$.

By comparing Theorems 1 and 3, we may examine the phrase 'solutions became flat' more closely. First of all, $B$ and $\mathfrak{B}$ featuring in (2.17) and (3.6) and defined in (2.18) and (3.5) are two realizations of the same operator in two different spaces (provided, as assumed here, that $c^{+}=c^{-}=0$ and $\alpha$ and $\beta$ are constants). Because of that the limit dynamics is in both cases the same. However, the operators $\mathcal{P}$ of (2.17) and $\mathcal{P}_{p, q}$ of (3.6) differ significantly (unless $p=q=0$ ). This is a reflection of the fact that Theorems 1 and 3 are results of different averaging mechanisms. As long as we are facing an nonadhesive membrane, functions are averaged by means of (2.19), but a sticky membrane leads to (3.7). In other words, our approximating scheme is robust in the sense that the limit process does not depend on the mechanism of filtering 
through the membrane. Nevertheless, the averaging that leads to this process does, and so does the limit: by changing $p$ and $q$ we change the initial condition for the limit master equation.

On the other hand, neither the limit semigroup nor the projection depend on the measures $\mu$ and $\nu$. It is thus irrelevant whether after filtering through the membrane a Brownian particle restarts it chaotic movement close to the vicinity of the membrane, on its other side (though this is the most natural choice) or somewhere further away. This effect is not surprising in view of the averaging property of diffusion, discussed briefly above.

\section{Proof of Theorem 3}

This section is devoted to a step-by-step proof of Theorem 3, intertwined with a similar proof of the generation result.

4.1. A building block: a holomorphic, Feller semigroup in $[0,1]$ and its asymptotic behavior

We start with a comment on notations: Sects. 4.1-4.3 are devoted to the vertical component of the main semigroup, and thus, we should think of the related functions (arguments of the semigroup) as depending on the $z$ variable. However, in the following analysis it will be more convenient to use $x$ as a variable instead. We will come back to using the coordinates of the previous section in Sect. 4.4.

Let $C[0,1]$ be the space of continuous functions on the unit interval $[0,1]$, and let $C^{2}[0,1]$ be its subspace composed of twice continuously differentiable functions. Moreover, let for any $r \in[0,1]$, the operator $G_{r}$ be given by

$$
G_{r} f=f^{\prime \prime}
$$

on the domain composed of $f \in C^{2}[0,1]$ such that

$$
r f^{\prime \prime}(0)-(1-r) f^{\prime}(0)=0 \text { and } f^{\prime}(1)=0 .
$$

As we shall see in this section, $G_{r}$ is a generator of a conservative Feller semigroup $\left(\mathrm{e}^{t G_{r}}\right)_{t \geq 0}$ (i.e., of a strongly continuous semigroup of positive contractions such that $\mathrm{e}^{t G_{r}} 1_{[0,1]}=1_{[0,1]}$, where $\left.1_{[0,1]}(x)=1, x \in[0,1]\right)$ in $C[0,1]$.

The process related to $G_{r}$ is a Brownian motion on $[0,1]$ with reflecting barrier at $x=1$ and a sticky barrier at $x=0$ (see [60, p. 127] or [17, pp. 19-20] and the references given there), trapping Brownian particles for 'an infinitely short time' (if $r \neq 1$ ). The duration of the imprisonment of the particles at $x=0$ depends on $r$ : for $r=1$ the particles are for ever trapped at $x=0$ and for $r=0$ they are reflected. For intermediate $r$ the set of times when a particle starting at $x=0$ is at $x=0$ again is of positive Lebesgue measure, and this measure increases with $r$ ([60, p. 128]). In what follows, $r$ will be referred to as a stickiness coefficient. 
As it transpires, as $t \rightarrow \infty$, a statistical equilibrium is reached between the particles trapped at $x=0$ and those evenly distributed across $[0,1]$ by diffusion. This fact is expressed in the following formula:

$$
\lim _{t \rightarrow \infty} \mathrm{e}^{t G_{r}} f=P_{r} f
$$

where

$$
P_{r} f(x)=r f(0)+(1-r) \int_{0}^{1} f(y) \mathrm{d} y, \quad x \in[0,1] .
$$

We prove (4.2) in Theorem 4, further down. We start our analysis with the generation result.

Proposition 2. The operator $G_{r}, r \in[0,1]$ is a conservative Feller generator.

Proof. The argument presented in [17, p. 17] shows that $G_{r}$ satisfies the positive maximum principle. It is also clear that $G_{r}$ is densely defined, that $1_{[0,1]}$ belongs to $D\left(G_{r}\right)$ and that $G_{r} 1_{[0,1]}=0$. Therefore, by the Hille-Yosida theorem for Feller semigroups ([36, Thm. 2.6, p. 13 and Thm. 2.2, p. 165], or [11, Thm. 8.3.4, p. 328]) it suffices to check the range condition: for any $g \in C[0,1]$ and $\lambda>0$ there is an $f \in D\left(G_{r}\right)$ such that

$$
\lambda f-G_{r} f=g .
$$

(In particular, existence of solutions to the resolvent equation for one $\lambda>0$ and all $g$ implies that $G_{r}$ is closed, see, e.g., [36] Lemma 2.2, p. 11.) To this end, we recall that $h=h_{\lambda, g}$ defined by

$$
h(x)=\frac{1}{2 \sqrt{\lambda}} \int_{0}^{1} \mathrm{e}^{-\sqrt{\lambda}|x-y|} g(y) \mathrm{d} y, \quad x \in[0,1],
$$

belongs to $C^{2}[0,1]$ and satisfies $\lambda h-h^{\prime \prime}=g$. Therefore, for any constant $C$, the same is true about $f \in C^{2}[0,1]$ given by

$$
f(x)=C \cosh \sqrt{\lambda}(1-x)-h(1) \sinh \sqrt{\lambda}(1-x)+h(x), \quad x \in[0,1] .
$$

Since $h^{\prime}(1)=-\sqrt{\lambda} h(1)$, we have $f^{\prime}(1)=0$. Moreover, the first condition in (4.1) is satisfied iff

$$
C=\frac{r[\lambda h(1) \sinh \sqrt{\lambda}+g(0)-\lambda h(0)]+(1-r)[h(1) \sqrt{\lambda} \cosh \sqrt{\lambda}+\sqrt{\lambda} h(0)]}{\lambda r \cosh \sqrt{\lambda}+(1-r) \sqrt{\lambda} \sinh \sqrt{\lambda}} .
$$

Since $f$ with so-defined $C$ belongs to $D\left(G_{r}\right)$, we are done.

For the study of the asymptotic behavior of the semigroups generated by $G_{r}, r \in$ $[0,1]$ we need a number of auxiliary results, presented below. The first of these reveals that each $\left(\mathrm{e}^{t G_{r}}\right)_{t \geq 0}$ is more regular than an ordinary Feller semigroup. 
Proposition 3. Operators $G_{r}, r \in[0,1]$ are generators of strongly continuous cosine families in $C[0,1]$.

Proof. This is a particular instance of theorems proved in [37,83,84]. Since the proofs of these general theorems are quite involved, and our case is rather simple, for completeness, we sketch a straightforward proof based on the method of images (see $[13,14])$. This method leads to a semi-explicit formula for the cosine family, and we will use this formula later.

Given a continuous function $f$ on $[0,1]$ we extend it to the interval $[0,2]$ by symmetry about $x=1$, by defining

$$
f(x)=f(2-x)
$$

for $x \in[1,2]$. If $f$ is twice continuously differentiable on $[0,1]$ and $f^{\prime}(1)=0$, the so-extended function is twice continuously differentiable on [0, 2]. Next, if $r \in[0,1)$, we extend $f$ to $[-2,2]$ by agreeing that (comp. [13], Eq. (2.4))

$$
f(-x)=2 \mathrm{e}^{-\kappa t} f(0)+\kappa \int_{0}^{x} \mathrm{e}^{-\kappa(x-y)} f(y) \mathrm{d} y-f(x),
$$

for $x \in[0,2]$, where $\kappa:=\frac{1-r}{r}$, and note that for $r=1$ this is an odd extension of $f$ : we have

$$
f(-x)=2 f(0)-f(x)
$$

(comp. [60, p. 125]). If $r=0$, we take $f(-x)=f(x)$ (symmetry about $x=0$ ). These extensions are chosen so that $f$ is twice continuously differentiable provided $f \in D\left(G_{r}\right)$ (see [13, pp. 667 and 674]). Having defined (an extension of) $f$ on [-2, 2] we may extend its definition to $[-2,4]$ by formula $(4.7)$, and then again to $[-4,4]$ by formula (4.8). Continuing this procedure of repeated reflections (see [39, p. 341] or [20]), we construct a twice continuously differentiable function on the entire line such that (4.7) and (4.8) are true for all $x \geq 1$ and all $x \geq 0$, respectively. This allows defining the family $\left(C_{r}(t)\right)_{t \in \mathbb{R}}$ of operators in $C[0,1]$ by

$$
C_{r}(t) f(x)=\frac{1}{2}(f(x+t)+f(x-t)), \quad x \in[0,1], t \in \mathbb{R} ;
$$

(note that the extension of $f$ depends on $r$ and so do these operators). The extension of $f$ is chosen in such a way that $C_{r}(t) f \in D\left(G_{r}\right)$ for all $t \in \mathbb{R}$ provided $f \in D\left(G_{r}\right)$. It follows that $\left(C_{r}(t)\right)_{t \in \mathbb{R}}$ is a cosine family (see [13] for details). Moreover, since for $f \in D\left(G_{r}\right)$, the extension of $f$ constructed above is twice continuously differentiable on $\mathbb{R}, \lim _{t \rightarrow 0} \frac{2\left(C_{r}(t) f-f\right)}{t^{2}}=f^{\prime \prime}$ for $f \in D\left(G_{r}\right)$. Therefore, the generator of $\left(C_{r}(t)\right)_{t \in \mathbb{R}}$ extends $G_{r}$. However, since (by Proposition 2) the range of $\lambda-G_{r}$ is the entire $C[0,1]$ no cosine family generator can be a proper extension of $G_{r}$, and we conclude that the generator of $\left(C_{r}(t)\right)_{t \in \mathbb{R}}$ is $G_{r}$.

Lemma 1. Let $r \in[0,1)$. Then, the semigroup $\left(\mathrm{e}^{t G_{r}}\right)_{t \geq 0}$ is irreducible: for any $\lambda>0$ and $g \geq 0$ the solution to the resolvent equation (4.4) is strictly positive. 
Proof. The idea lying behind the following proof is that the transition probabilities of the process related to $\left(\mathrm{e}^{t G_{r}}\right)_{t \geq 0}$ are larger than those of the minimal process in which a particle reaching $x=0$ is killed and removed from the state space. Nevertheless, the argument is purely 'analytic.'

As a bit of algebra shows,

$$
\frac{r[x h(1) \sinh x-x h(0)]+(1-r)[h(1) \cosh x+h(0)]}{x r \cosh x+(1-r) \sinh x}>\frac{h(1) \sinh x-h(0)}{\cosh x},
$$

for all $x>0$ and $r \in[0,1)$ (for $r=1$ this turns into equality). Hence, even if $g(0)=0, f$ defined by (4.5) and (4.6) satisfies $f(0)>0$. By the same token, $C$ of (4.6) is larger than

$$
C_{0}:=\frac{h(1) \sinh \sqrt{\lambda}-h(0)}{\cosh \sqrt{\lambda}},
$$

and thus, $f$ is larger than $f_{0}$, where $f_{0}$ is defined by (4.5) with $C$ replaced by $C_{0}$.

To show that $f_{0}(x)>0$ for all $x \in(0,1]$ we first note that

$$
f_{0}(x)=\frac{\sinh \sqrt{\lambda} x}{\cosh \sqrt{\lambda}} h(1)-\frac{\cosh \sqrt{\lambda}(1-x)}{\cosh \sqrt{\lambda}} h(0)+h(x) .
$$

By the definition of $h$, it follows that

$$
f_{0}(x)=\frac{1}{4 \sqrt{\lambda} \cosh \sqrt{\lambda}} \int_{0}^{1} k_{\lambda}(x, y) g(y) \mathrm{d} y,
$$

where

$$
\begin{aligned}
k_{\lambda}(x, y)= & 2 \cosh \sqrt{\lambda} \mathrm{e}^{-\sqrt{\lambda}|x-y|}+2 \sinh \sqrt{\lambda} x \mathrm{e}^{\sqrt{\lambda}(y-1)} \\
& -2 \cosh \sqrt{\lambda}(1-x) \mathrm{e}^{-\sqrt{\lambda} y} \\
= & \mathrm{e}^{-\sqrt{\lambda}(|x-y|+1)}+\mathrm{e}^{-\sqrt{\lambda}(|x-y|-1)}+\mathrm{e}^{\sqrt{\lambda}(x+y-1)} \\
& -\mathrm{e}^{\sqrt{\lambda}(y-x-1)}-\mathrm{e}^{\sqrt{\lambda}(1-x-y)}-\mathrm{e}^{\sqrt{\lambda}(x-1-y)} .
\end{aligned}
$$

For $y \leq x$, this expression reduces to

$$
\mathrm{e}^{\sqrt{\lambda}(1-x+y)}+\mathrm{e}^{\sqrt{\lambda}(x+y-1)}-\mathrm{e}^{\sqrt{\lambda}(1-x-y)}-\mathrm{e}^{\sqrt{\lambda}(x-1-y)} \geq 0
$$

with equality holding only if $y=0$. Analogously, for $0<x<y \leq 1$, this reduces to

$$
\mathrm{e}^{\sqrt{\lambda}(1-y+x)}+\mathrm{e}^{\sqrt{\lambda}(x+y-1)}-\mathrm{e}^{\sqrt{\lambda}(y-x-1)}-\mathrm{e}^{\sqrt{\lambda}(1-x-y)}>0
$$

(since $x>0)$. This shows that for each $x \in(0,1]$ the function $(0,1] \ni y \mapsto k_{\lambda}(x, y)$ is continuous and strictly positive. Therefore, $f_{0}(x)>0$ for $x \in(0,1]$, and the proof is complete. 
Lemma 2. The domain $D\left(G_{r}\right)$, when equipped with the graph norm $\|f\|_{G_{r}}=\|f\|+$ $\left\|f^{\prime \prime}\right\|$ where $\|\cdot\|$ is the norm in $C[0,1]$, embeds compactly into $C[0,1]$.

Proof. We are to prove that the unit ball in $D\left(G_{r}\right)$, when considered as a subset of $C[0,1]$ is relatively compact. To this end, we note that members $f$ of this ball satisfy $\|f\|+\left\|f^{\prime \prime}\right\| \leq 1 \quad$ and $\quad f(x)=f(0)+f^{\prime}(0) x+\int_{0}^{x} \int_{0}^{y} f^{\prime \prime}(z) \mathrm{d} z \mathrm{~d} y, x \in[0,1]$ where $f^{\prime}(0)=-\int_{0}^{1} f^{\prime \prime}(y) \mathrm{d} y$ (by the second part of the boundary conditions (4.1)). It follows that $\left|f^{\prime}(0)\right| \leq 1$ and then that $|f(x)-f(y)| \leq 2|x-y|, x, y \in[0,1]$, and thus, these functions are equicontinuous. Hence, we are done by the Arzelá-Ascoli Theorem.

Theorem 4. There are positive constants $K$ and $\omega$ (depending perhaps on $r$ ) such that

$$
\left\|\mathrm{e}^{t G_{r}}-P_{r}\right\| \leq K \mathrm{e}^{-\omega t}, \quad t \geq 0
$$

where $P_{r}$ is defined in (4.3).

Proof. (i) The case $r=0$ is well known (see, e.g., [17, pp. 177-180]).

(ii) The case $r \in(0,1)$. Since $D\left(G_{r}\right)$ embeds compactly into $C[0,1]$ (by Lemma 2), the resolvent operators $\left(\lambda-G_{r}\right)^{-1}, \lambda>0$ are compact ([35, p. 117]). Also, since $G_{r}$ generates a cosine family (by Proposition 3 ), the Weierstrass formula implies that $\left(\mathrm{e}^{t G_{r}}\right)_{t \geq 0}$ may be extended to a holomorphic semigroup (of angle $\pi / 2$, in the space of complex functions on [0, 1])—see, e.g., [5, pp. 219-220]. It follows that $\left(\mathrm{e}^{t G_{r}}\right)_{t \geq 0}$ is immediately norm continuous (i.e., $\lim _{s \rightarrow t} \| \mathrm{e}^{s G_{r}}-$ $\mathrm{e}^{t G_{r}} \|=0, t>0$ )— this may be seen, e.g., by combining Lemma 4.2 p. 52 and Theorem 5.2 (point (d)) p. 62 in [69]. This together with compactness of the resolvent operators implies that also $\mathrm{e}^{t G_{r}}, t>0$ are compact (see [35, p. 117] or [69, p. 48]). Finally, by Lemma 2, $\left(\mathrm{e}^{t G_{r}}\right)_{t \geq 0}$ is irreducible. Therefore, all assumptions of the theorem in Sect. 3.5.1 of [3] are satisfied. It follows that (i) the spectral bound

$$
s\left(G_{r}\right)=\sup \left\{\Re \lambda: \lambda \in \sigma\left(G_{r}\right)\right\}
$$

where $\sigma\left(G_{r}\right)$ is the spectrum of $G_{r}$, is larger than $-\infty$, and (ii) there are positive constants $K$ and $\omega$ and a nonzero operator $P_{r}$ such that

$$
\left\|\mathrm{e}^{-s\left(G_{r}\right) t} \mathrm{e}^{t G_{r}}-P_{r}\right\| \leq K \mathrm{e}^{-\omega t}, \quad t \geq 0 .
$$

Since $\left(\mathrm{e}^{t G_{r}}\right)_{t \geq 0}$ is a contraction semigroup, $s\left(G_{r}\right) \leq 0$, and since $\mathrm{e}^{t G_{r}} 1_{[0,1]}=$ $1_{[0,1]}, s\left(G_{r}\right)$ cannot be strictly negative. Hence, $s\left(G_{r}\right)=0$ and to prove (4.10) we only need to show that $P_{r}$ in (4.11) is given by (4.3). To this end, recall that existence of the $\operatorname{limit}_{\lim _{t \rightarrow \infty}} \mathrm{e}^{t G_{r}} g, g \in C[0,1]$ implies existence of 
$\lim _{\lambda \rightarrow 0} \lambda\left(\lambda-G_{r}\right)^{-1} g$ and the two then coincide. Moreover, a limit in the norm, when it exists, must of course coincide with the pointwise limit. On the other hand, $\lim _{\lambda \rightarrow 0} \lambda\left(\lambda-G_{r}\right)^{-1} g(x)$ is easy to calculate, since we know the exact form of $f(x)=\left(\lambda-G_{r}\right)^{-1} g(x)$; it is given in (4.4) and (4.5). Namely, it is easy to see that $\lim _{\lambda \rightarrow 0} \lambda\left(\lambda-G_{r}\right)^{-1} g(x)=\lim _{\lambda \rightarrow 0} \lambda C$ for the $C=C_{\lambda, g}$ of (4.5). Moreover, when multiplied by $\lambda$ this $C$ converges to $r g(0)+(1-r) \int_{0}^{1} g(x) \mathrm{d} x$, as $\lambda \rightarrow 0$ (note that $h$ appearing in the definition of $C$ also depends on $\lambda$ ). This completes the proof.

(iii) In the case $r=1$, the semigroup $\left(\mathrm{e}^{t G_{r}}\right)_{t \geq 0}$ is not irreducible (see the proof of Lemma 1 -the solution $f$ to the resolvent equation equals 0 at $x=0$ as long as $g(0)=0$ ), and we need to proceed differently. Fortunately, the very fact that $\left(\mathrm{e}^{t G_{1}}\right)_{t \geq 0}$ is not irreducible suggests a different line of attack. The cosine family $\left(C_{1}(\bar{t})\right)_{t \in \mathbb{R}}$ constructed in Proposition 3 leaves the subspace $C_{0}(0,1]=$ $\{f \in C[0,1] ; f(0)=0\}$ of $C[0,1]$ invariant: if $f(0)=0$ then $C_{1}(t) f(0)=$ 0 for all $t \in \mathbb{R}$, because the graph of the extension of $f$ featuring in (4.9) is antisymmetric about $x=0$. The generator, say $G_{1}^{0}$, of the restriction of $\left(C_{1}(t)\right)_{t \in \mathbb{R}}$ (and of the restriction of $\left.\left(\mathrm{e}^{t G_{1}}\right)_{t \geq 0}\right)$ to $C_{0}(0,1]$ is the part of $G_{1}$ in this subspace, i.e., $G_{1}^{0}$ is the operator of the second derivative on the domain $D\left(G_{1}^{0}\right)=\left\{f \in C_{0}(0,1] \cap C^{2}[0,1], f^{\prime \prime} \in C_{0}(0,1]\right\}$ or, equivalently, $D\left(G_{1}^{0}\right)=$ $\left\{f \in C_{0}(0,1] \cap C^{2}[0,1], f^{\prime \prime}(0)=0\right\}$. Also, for any $g \in C_{0}(0,1]$ the function $f(x)=\int_{0}^{x} \int_{0}^{y} g(z) \mathrm{d} z \mathrm{~d} y, x \in[0,1]$ belongs to $D\left(G_{1}^{0}\right)$ and we have $G_{1}^{0} f=g$. It follows that 0 belongs to the resolvent set of $G_{1}^{0}$, and, since $\left(\mathrm{e}^{t G_{1}^{0}}\right)_{t \geq 0}$ is a positive contraction semigroup, Proposition 3.11.2 in [5] implies that $s\left(G_{1}^{0}\right)<0$. Therefore, see [3, p. 13], there are positive constants $K$ and $\omega$ such that

$$
\left\|\mathrm{e}^{t G_{1}^{0}}\right\|_{\mathcal{L}\left(C_{0}(0,1]\right)} \leq K \mathrm{e}^{-\omega t}, \quad t \geq 0 .
$$

On the other hand, $\left(\mathrm{e}^{t G_{1}}\right)_{t \geq 0}$ being conservative, given $f \in C[0,1]$ we may consider $f_{0}:=f-f(0) 1_{[0,1]} \in C_{0}(0,1]$ and write

$$
\left\|\mathrm{e}^{t G_{1}} f-f(0) 1_{[0,1]}\right\|=\left\|\mathrm{e}^{t G_{1}} f_{0}\right\|=\left\|\mathrm{e}^{t G_{1}^{0}} f_{0}\right\| \leq K \mathrm{e}^{-\omega t}\left\|f_{0}\right\| \leq 2 K \mathrm{e}^{-\omega t}\|f\| ;
$$

the first equality here is a consequence of $\mathrm{e}^{t G_{1}} 1_{[0,1]}=1_{[0,1]}$ combined with the definition of $f_{0}$. This completes the proof.

Before completing this section, we note that $C[0,1]$ is isometrically isomorphic to $C[-1,0]$, the space of continuous functions on $[-1,0]$, with positivity preserving isometric isomorphism $I: C[-1,0] \rightarrow C[0,1]$ given by $\operatorname{If}(x)=f(-x), x \in[0,1]$. The operator $G_{r}^{I}$ given by $G_{r}^{I} f=f^{\prime \prime}$ on the domain composed of twice continuously differentiable functions $f \in C[-1,0]$ such that

$$
r f^{\prime \prime}(0)+(1-r) f^{\prime}(0)=0 \text { and } f^{\prime}(-1)=0
$$

is the image of $G_{r}$ in $C[-1,0]$. This is to say that $f$ belongs to $D\left(G_{r}^{I}\right)$ iff If belongs to $D\left(G_{r}\right)$ and we have $G_{r} I f=I G_{r}^{I} f$. It follows that $\left(\mathrm{e}^{t G_{r}^{I}}\right)_{t \geq 0}$ mirrors properties 
of $\left(\mathrm{e}^{t G_{r}}\right)_{t \geq 0}$. Therefore, as a corollary to Proposition 2 and Theorem 4 we obtain the following result.

Theorem 5. For any $r \in[0,1]$, the operator $G_{r}^{I}$ is a Feller generator in $C[-1,0]$. Moreover, there are positive constants $K$ and $\omega$ (depending perhaps on $r$ ) such that

$$
\left\|\mathrm{e}^{t G_{r}^{I}}-P_{r}^{I}\right\| \leq K \mathrm{e}^{-\omega t}, \quad t \geq 0
$$

where $P_{r}^{I}=I^{-1} P_{r} I$ for $P_{r}$ defined in (4.3), i.e.,

$$
P_{r}^{I} f(x)=r f(0)+(1-r) \int_{-1}^{0} f(y) \mathrm{d} y, \quad x \in[-1,0] .
$$

4.2. The vertical component: a generation theorem

4.2.1. Sticky membrane at $x=0$; no communication between the intervals $[-1,0-]$ and $[0+, 1]$

Let $C(U)$ be the space of continuous functions on the union $U$ of two unit intervals $U:=[-1,0-] \cup[0+, 1]$. Here, similarly as before, we imagine that there is an infinitely thin membrane at $x=0$ and think of $0-$ and $0+$ as the points to the immediate left and to the immediate right of this membrane, respectively.

Each element $f$ of $C(U)$ may be thought of as the sum of $f_{1} \in C[-1,0]$ and $f_{2} \in C[0,1]$ defined by

$$
f_{1}:=f_{\mid[-1,0-]} \text { and } f_{2}:=f_{\mid[0+, 1]} .
$$

This is to say that $C(U)$ is a direct sum of its two subspaces which may be identified with $C[-1,0]$ and $C[0,1]$.

With this convention, given $p, q \in[0,1]$, it makes sense to define

$$
T(t) f=\mathrm{e}^{t G_{p}^{I}} f_{1}+\mathrm{e}^{t G_{q}} f_{2}
$$

where $G_{p}^{I}$ and $G_{q}$ are defined in Sect. 4.1. It is clear that $(T(t))_{t \geq 0}$ is a conservative Feller semigroup in $C(U)$ and that its generator, say $A_{0}$, is defined on the domain composed of $f$ such that $f_{1} \in D\left(G_{p}^{I}\right)$ and $f_{2} \in D\left(G_{q}\right)$ by the formula

$$
A_{0} f=G_{p}^{I} f_{1}+G_{q} f_{2}=f_{1}^{\prime \prime}+f_{2}^{\prime \prime} .
$$

In other words, an $f$ belongs to $D\left(A_{0}\right)$ iff when restricted to either of the two subintervals forming $U$ it is twice continuously differentiable and the following boundary conditions are satisfied:

$$
\begin{aligned}
f^{\prime}(-1)=f^{\prime}(1) & =0, \\
p f^{\prime \prime}(0-)+(1-p) f^{\prime}(0-) & =0, \\
q f^{\prime \prime}(0+)-(1-q) f^{\prime}(0+) & =0 .
\end{aligned}
$$


Moreover, $A_{0} f=f^{\prime \prime}$. This operator describes two independent Brownian motions in two non-communicating intervals. In the right interval, the related process is a Brownian motion with reflecting barrier at $x=1$ and a sticky barrier at $0+$ with stickiness coefficient $q$. In [-1,0-], the process is a mirror image of an analogous Brownian motion with stickiness coefficient $p$.

As a corollary to Theorems 4 and 5, we also have the following information on the asymptotic behavior of the semigroup/process under consideration.

Theorem 6. There are positive constants $K$ and $\omega$ (depending perhaps on $p$ and $q$ ) such that

$$
\left\|\mathrm{e}^{t A_{0}}-P_{p, q}\right\| \leq K \mathrm{e}^{-\omega t}, \quad t \geq 0,
$$

where $\mathrm{e}^{t A_{0}}=T(t)$ and $P_{p, q}$ is given by $P_{p, q} f=\left(P_{p}^{I} f_{1}, P_{q} f_{2}\right)$.

\subsubsection{A Greiner-like perturbation of the generator leading to communication between the intervals}

Let $A$ in $C(U)$ be defined as follows. Its domain is composed of $f \in C(U)$ such that $f_{1} \in C^{2}[-1,0], f_{2} \in C^{2}[0,1]$ and $f^{\prime}(1)=f^{\prime}(-1)=0$. Also,

$$
A f=f_{1}^{\prime \prime}+f_{2}^{\prime \prime}=f^{\prime \prime} \text {. }
$$

Next, let $L: D(A) \rightarrow \mathbb{R}^{2}$ be defined by

$$
\begin{array}{r}
L f=\left(p f^{\prime \prime}(0-)+(1-p) f^{\prime}(0-),\right. \\
\left.q f^{\prime \prime}(0+)-(1-q) f^{\prime}(0+)\right),
\end{array}
$$

so that, in particular, we see that $A_{0}$ of the previous subsection is $A$ restricted to ker $L$.

Let $\alpha$ and $\beta$ be non-negative numbers, let $\mu$ be a Borel probability measure on $[-1,0]$ and let $v$ be a Borel probability measure on $[0,1]$. Given such data, we define $\Phi: C(U) \rightarrow \mathbb{R}^{2}$ by

$$
\Phi f=(\alpha[v(f)-f(0-)], \beta[\mu(f)-f(0+)]), \quad f \in C(U) .
$$

Here and in what follows, for $f \in C(U)$, we write $\mu(f)$ and $\nu(f)$ to denote $\int_{-1}^{0} f_{1} \mathrm{~d} \mu$ and $\int_{0}^{1} f_{2} \mathrm{~d} \nu$, respectively. Our goal in this subsection is to show that the operator

$$
A_{\Phi}:=A_{\mid D\left(A_{\Phi}\right)},
$$

i.e., the operator $A$ restricted to

$$
D\left(A_{\Phi}\right):=\{f \in D(A) ; L f=\Phi f\}
$$

is a conservative Feller generator.

In the related process, communication between the intervals $[-1,0-]$ and $[0+, 1]$ is possible through a semi-permeable membrane at $x=0$. A particle starting in $[0+, 1]$ 
performs a sticky Brownian motion in this interval (with reflecting barrier at $x=1$ ), but the time it spends at the sticky boundary $x=0^{+}$is measured (for $r=0$ this measuring is done by the Lévy local time for Brownian motion, see [50,60]). After a sufficiently long random time is spent at the boundary, the particle filters through the membrane to its other side. The larger is $\beta$ the shorter is the time needed to filter through the membrane, and thus, it is appropriate to refer to $\beta$ as the permeability coefficient. In particular, for $r=1$, the time spent at the boundary is exponential with parameter $\beta$; in agreement with [38, p. 3], this process will be called an elementary jump process. For $r=0$, the time spent at the boundary is exponential (with the same parameter) with respect to the Lévy local time; such processes are termed snapping out Brownian motions by Lejay [59]. The measure $\mu$ describes the particle's position after it filters through the membrane. Intuitively, the most natural choice seems to be $\mu(f)=f(0-)$ (the Dirac measure concentrated at $x=0-$ ), describing the situation where the particle after filtering through the membrane starts its motion from the closest vicinity of $x=0$. However, for $p=q=1$ (i.e., in the case of elementary jump through the membrane) this leads to a rather uninteresting dynamics, and thus, we decided to work with a more general $\mu$. Needless to say, in $[-1,0-]$ the process is a mirror reflection of a similar Brownian motion with stickiness coefficient $p$ and permeability coefficient $\alpha$.

We note that $A_{0}$ and $A_{\Phi}$ are restrictions of the same operator to different domains. Hence, in proving the following generation theorem we use the seminal ideas of Greiner [43], who pioneered the research on domain changing perturbations of semigroups' generators. (See also [65] for an interesting perspective on Greiner's result.)

Theorem 7. The operator $A_{\Phi}$ is a conservative Feller generator.

Proof. As in [17, p. 17] it can be shown that $A_{\Phi}$ satisfies the positive maximum principle. Moreover, it is clear that $1_{U} \in D\left(A_{\Phi}\right)$ with $A_{\Phi} 1_{U}=0$ (where, of course, $1_{U}(x)=1$ for $\left.x \in U\right)$. Since $A_{\Phi}$ is also densely defined, we will be done once existence of an $f \in D\left(A_{\Phi}\right)$ satisfying

$$
\lambda f-A_{\Phi} f=g
$$

is established for a fixed $\lambda>0$ and all $g \in C(U)$.

This is where we follow the approach of Greiner. First, we note that the kernel of $\lambda-A$ of is spanned by $k_{1, \lambda} \in C[-1,0]$ and $k_{2, \lambda} \in C[0,1]$ defined by

$$
\begin{array}{ll}
k_{1, \lambda}(x)=\cosh \sqrt{\lambda}(x+1), & x \in[-1,0], \\
k_{2, \lambda}(x)=\cosh \sqrt{\lambda}(x-1), & x \in[0,1] .
\end{array}
$$

For an $f=C k_{1, \lambda}+D k_{2, \lambda}$ in this kernel ( $C$ and $D$ are real constants),

$$
L f=\left(C m_{\lambda}(p), D m_{\lambda}(q)\right)
$$

where

$$
m_{\lambda}(r)=r \lambda \cosh \sqrt{\lambda}+(1-r) \sqrt{\lambda} \sinh \sqrt{\lambda} .
$$


Thus, $L$ establishes a one-to-one correspondence between $\operatorname{ker}(\lambda-A)$ and $\mathbb{R}^{2}$ with $L_{\lambda}:=\left(L_{\operatorname{ker}(\lambda-A)}\right)^{-1}, L_{\lambda}: \mathbb{R}^{2} \rightarrow \operatorname{ker}(\lambda-A)$ given by

$$
L_{\lambda}\left(x_{1}, x_{2}\right)=\frac{x_{1}}{m_{\lambda}(p)} k_{1, \lambda}+\frac{x_{2}}{m_{\lambda}(q)} k_{2, \lambda} .
$$

We note that

$$
\left\|L_{\lambda}\right\| \leq \max _{r=p, q} \frac{\cosh \sqrt{\lambda}}{m_{\lambda}(r)}
$$

(here, $\mathbb{R}^{2}$ is equipped with the max norm). Also,

$$
\frac{\cosh \sqrt{\lambda}}{m_{\lambda}(r)} \leq \begin{cases}\frac{1}{r \lambda}, & r>0, \lambda>0 \\ \frac{\cosh \sqrt{\lambda}}{\sqrt{\lambda} \sinh \sqrt{\lambda}} \leq \frac{M_{0}}{\sqrt{\lambda}}, & r=0, \lambda>1\end{cases}
$$

where $M_{0}:=\sup _{x \geq 1} \frac{\cosh x}{\sinh x}$ is finite, since the function involved here is continuous and has finite limits at $x=1$ and $x=\infty$. It follows that for sufficiently large $\lambda$ the map $L_{\lambda} \Phi$ has norm smaller than 1 , and thus, $I_{C(U)}-L_{\lambda} \Phi$ is invertible.

Consider such a $\lambda$ and a $g \in C(U)$. Let

$$
f:=\left(I_{C(U)}-L_{\lambda} \Phi\right)^{-1}\left(\lambda-A_{0}\right)^{-1} g
$$

so that $f=L_{\lambda} \Phi f+\left(\lambda-A_{0}\right)^{-1} g$ (comp. [43] Lemma 1.4). Since $L_{\lambda} \Phi f \in \operatorname{ker}(\lambda-$ $A) \subset D(A)$ and $\left(\lambda-A_{0}\right)^{-1} g \in D(A)$, we see that $f \in D(A)$. Then, the calculation $L f=L L_{\lambda} \Phi f+L\left(\lambda-A_{0}\right)^{-1} g=\Phi f+0=\Phi f$, shows that $f$ belongs to $D\left(A_{\Phi}\right)$. Moreover, since $A\left(\lambda-A_{0}\right)^{-1} g=A_{0}\left(\lambda-A_{0}\right)^{-1} g=\lambda\left(\lambda-A_{0}\right)^{-1} g-g$ and $L_{\lambda} \Phi f$ belongs to $\operatorname{ker}(\lambda-A)$,

$$
\begin{aligned}
A_{\Phi} f=A f & =A\left(L_{\lambda} \Phi f+\left(\lambda-A_{0}\right)^{-1} g\right)=\lambda L_{\lambda} \Phi f+\lambda\left(\lambda-A_{0}\right)^{-1} g-g \\
& =\lambda f-g,
\end{aligned}
$$

proving that $f$ solves the resolvent equation (4.16).

We note that, since operators satisfying the positive maximum principle are dissipative (see, e.g., [36] Lemma 2.1, p. 165), the solution to the resolvent equation is unique. Hence, as a by-product of the proof, we obtain

$$
\left(\lambda-A_{\Phi}\right)^{-1}=\left(I_{C(U)}-L_{\lambda} \Phi\right)^{-1}\left(\lambda-A_{0}\right)^{-1},
$$

for all $\lambda>0$ such that $I_{C(U)}-L_{\lambda} \Phi$ is invertible (this is in fact repeating Lemma 1.4 in [43] in the context of Feller generators).

\subsection{The vertical component: a limit theorem}

Before continuing, we recall that the classical Trotter-Kato Theorem (see, e.g., $[42,69])$ says that strongly continuous equibounded semigroups $\left(\mathrm{e}^{t A^{\varepsilon}}\right)_{t \geq 0}, \varepsilon \in(0,1]$ 
in a Banach space $E$ converge as $\varepsilon \rightarrow 0$ to a strongly continuous semigroup $\left(\mathrm{e}^{t B}\right)_{t \geq 0}$, i.e.,

$$
\lim _{\varepsilon \rightarrow 0} \mathrm{e}^{t A^{\varepsilon}} f=\mathrm{e}^{t B} f, \quad t \geq 0, f \in E,
$$

iff

$$
\lim _{\varepsilon \rightarrow 0}\left(\lambda-A^{\varepsilon}\right)^{-1} f=(\lambda-B)^{-1} f, \quad f \in E,
$$

for some/all $\lambda>0$; moreover, then the limit (4.21) is uniform in $t$ in compact subsets of $[0, \infty)$. In other words, such regular convergence of semigroups is completely characterized (see also $[5,11,17,36]$ for the Sova-Kurtz version $[55,81]$ of this characterization).

However, in the theory of singular perturbations and in the particular example we are studying here the limit semigroup is strongly continuous only on a subspace of $E$ : we are facing a limit theorem of the form

$$
\lim _{\varepsilon \rightarrow 0} \mathrm{e}^{t A^{\varepsilon}} f=\mathrm{e}^{t B} P f, \quad t>0, f \in E
$$

where $\left(\mathrm{e}^{t B}\right)_{t \geq 0}$ is a strongly continuous semigroup on a subspace $E_{0}$ of $E$ and $P$ is a projection on $E_{0}$ (in the sense that $P^{2}=P$ and $P f=f, f \in E_{0}$ ). Needless to say, in this case the classical theory does not work and, in particular, condition

$$
\lim _{\varepsilon \rightarrow 0}\left(\lambda-A^{\varepsilon}\right)^{-1} f=(\lambda-B)^{-1} P f, \quad f \in E,
$$

for all (some) $\lambda>0$ is necessary but not sufficient for (4.22) (see [10] or [17]).

As we have seen in Sect. 2, (4.23) may imply (4.22) provided that the semigroups involved possess additional regularity properties (like, for example, uniform holomorphicity—see, e.g., [17], Chapters 31 and 41 for details). A different set of conditions guaranteeing that (4.23) implies (4.22) has been given by T. G. Kurtz $[36,56,57]$. While Kurtz's singular convergence theorem is usually expressed in terms of the so-called extended limit of generators, for our subsequent analysis the following resolvent-version will be more practical. This result may be easily deduced, e.g., from combined Lemma 7.1 and Theorem 42.2 in [17].

Theorem 8. Suppose $A^{\varepsilon}, \varepsilon \in(0,1]$ are generators of strongly continuous equibounded semigroups. Suppose also that for some $\lambda>0$

$$
\lim _{\varepsilon \rightarrow 0}\left(\lambda-\varepsilon^{2} A^{\varepsilon}\right)^{-1}=\left(\lambda-A_{0}\right)^{-1}
$$

where $A_{0}$ is the generator of a strongly continuous semigroup $\left(\mathrm{e}^{t A_{0}}\right)_{t \geq 0}$ such that

$$
\text { Pf }:=\lim _{t \rightarrow \infty} \mathrm{e}^{t A_{0}} f, \quad f \in E
$$

exists. Then, condition (4.23) (for some $\lambda>0$, with the same P) implies (4.22), and the limit is uniform in $t$ in compact subsets of $(0, \infty)$; for $f \in E_{0}$ the limit is uniform in $t$ in compact subsets of $[0, \infty)$. 
We will apply this theorem to the Feller generators

$$
A^{\varepsilon}:=\varepsilon^{-2} A_{\varepsilon^{2} \Phi}
$$

In other words, we will study the situation in which diffusion is very fast while permeability coefficients of the membrane are small.

Let $E_{0} \subset C(U)$ be the subspace composed of functions which are constant in each of the subintervals forming $U$ (i.e., for $f \in E_{0}$ both $f_{1}$ and $f_{2}$ are constant functions). Each member of $E_{0}$ may be naturally identified with two real numbers, say $f^{-}$and $f^{+}$, and $E_{0}$ may be naturally identified with $\mathbb{R}^{2}$ with the maximum norm.

Theorem 9. Let $B$ be the operator in $E_{0}$ which may be identified with the matrix of (2.4). In other words, $B\left(f^{-}, f^{+}\right)=\left(\alpha\left(f^{+}-f^{-}\right), \beta\left(f^{-}-f^{+}\right)\right)$. Then,

$$
\lim _{\varepsilon \rightarrow 0} \mathrm{e}^{t A^{\varepsilon}} f=\mathrm{e}^{t B} P_{p, q} f, \quad t>0, f \in C(U),
$$

where $P_{p, q}$ is defined in Theorem 6 and the limit is uniform in $t$ in compact subsets of $(0, \infty)$; for $f \in E_{0}$ the limit is uniform in compact subsets of $[0, \infty)$.

For the proof of this result, we need the following lemma.

Lemma 3. For sufficiently large $\lambda$,

$$
\lim _{\varepsilon \rightarrow 0}\left(\lambda-A_{\varepsilon}\right)^{-1} f=(\lambda-B)^{-1} P_{p, q} f, \quad f \in C(U) .
$$

Proof. Solving the resolvent equation for $A^{\varepsilon}: \lambda f-A^{\varepsilon} f=g$ is equivalent to solving the resolvent equation for $A_{\varepsilon^{2} \Phi}$ with $\lambda$ replaced by $\varepsilon^{2} \lambda$ and $g$ replaced by $\varepsilon^{2} g$. On the other hand, by (4.19),

$$
\frac{\varepsilon^{2} \cosh \varepsilon \sqrt{\lambda}}{m_{\varepsilon^{2} \lambda}(r)} \leq \frac{1}{r \lambda}, \quad r \in(0,1], \lambda>0, \varepsilon \in(0,1] .
$$

Moreover,

$$
\frac{\varepsilon^{2} \cosh \varepsilon \sqrt{\lambda}}{m_{\varepsilon^{2} \lambda}(0)}=\frac{\varepsilon \cosh \varepsilon \sqrt{\lambda}}{\sqrt{\lambda} \sinh \varepsilon \sqrt{\lambda}} \leq\left\{\begin{array}{ll}
\frac{\varepsilon M_{2}}{\sqrt{\lambda}} \leq \frac{M_{2}}{\sqrt{\lambda}}, & \varepsilon \sqrt{\lambda} \geq 1, \\
\frac{M_{3}}{\lambda}, & \varepsilon \sqrt{\lambda} \in(0,1],
\end{array} \quad \varepsilon \in(0,1],\right.
$$

where $M_{2}:=\sup _{x>0} \frac{\cosh x}{\sinh x}$ and $M_{3}:=\sup _{x \in(0,1]} \frac{x \cosh x}{\sinh x}$ are finite because the functions $x \mapsto \frac{\cosh x}{\sinh x}$ and $x \mapsto \frac{x \cosh x}{\sinh x}$ are continuous and have finite limits at appropriate intervals' ends.

It follows, by (4.18), that for sufficiently large $\lambda$ the norm of $\varepsilon^{2} L_{\varepsilon^{2} \lambda} \Phi$ is smaller than 1 , regardless of the choice of $\varepsilon$, and so $I-\varepsilon^{2} L_{\varepsilon^{2} \lambda} \Phi$ is invertible for all $\varepsilon \in(0,1)$. Therefore, for such $\lambda$, by (4.20),

$$
\left(\lambda-A^{\varepsilon}\right)^{-1}=\varepsilon^{2}\left(\varepsilon^{2} \lambda-A_{\varepsilon^{2} \Phi}\right)^{-1}=\varepsilon^{2}\left(I_{C(U)}-L_{\varepsilon^{2} \lambda} \varepsilon^{2} \Phi\right)^{-1}\left(\varepsilon^{2} \lambda-A_{0}\right)^{-1} .
$$


Next, by Theorem $6, \lim _{\varepsilon \rightarrow 0} \varepsilon^{2}\left(\varepsilon^{2} \lambda-A_{0}\right)^{-1}=\lambda^{-1} P_{p, q}$, and we are left with analyzing the factor $\left(I_{C(U)}-L_{\varepsilon^{2} \lambda} \varepsilon^{2} \Phi\right)^{-1}$. To this end, we observe that for $f \in C(U)$ (see (4.17) and the definition of $\Phi$ )

$$
L_{\varepsilon^{2} \lambda} \varepsilon^{2} \Phi f=\frac{\varepsilon^{2} \alpha[v(f)-f(0-)]}{m_{\varepsilon^{2} \lambda}(p)} k_{1, \varepsilon^{2} \lambda}+\frac{\varepsilon^{2} \beta[f(0+)-\mu(f)]}{m_{\varepsilon^{2} \lambda}(q)} k_{1, \varepsilon^{2} \lambda}
$$

converges, as $\varepsilon \rightarrow 0$, to

$$
\begin{aligned}
\frac{\alpha[v(f)-f(0-)]}{\lambda} 1_{[-1,0]} & +\frac{\beta[\mu(f)-f(0+)]}{\lambda} 1_{[0,1]} \\
& =\lambda^{-1}(\alpha[v(f)-f(0-)], \beta[\mu(f)-f(0+)]),
\end{aligned}
$$

because, as it is easy to check, $\lim _{\varepsilon \rightarrow 0} \frac{\varepsilon^{2}}{m_{\varepsilon^{2} \lambda}(r)}=\lambda^{-1}, r \in[0,1]$. Since $\mu$ and $v$ are probability measures, for $f=\left(f^{-}, f^{+}\right)$in $E_{0}$, the latter vector is

$$
\lambda^{-1}\left(\alpha\left(f^{+}-f^{-}\right), \beta\left(f^{-}-f^{+}\right)\right)=\lambda^{-1} B\left(f^{-}, f^{+}\right) .
$$

This shows that

$$
\lim _{\varepsilon \rightarrow 0}\left(\lambda-A^{\varepsilon}\right)^{-1} f=\left(I_{C(U)}-\lambda^{-1} B\right)^{-1} \lambda^{-1} P_{p, q} f=(\lambda-B)^{-1} P_{p, q} f,
$$

as claimed.

Proof of Theorem 9. A similar (but simpler) analysis to that presented in Lemma 3 shows that

$$
\lim _{\varepsilon \rightarrow 0}\left(\lambda-\varepsilon^{2} A^{\varepsilon}\right)^{-1}=\lim _{\varepsilon \rightarrow 0}\left(\lambda-A_{\varepsilon^{2} \Phi}\right)^{-1}=\left(\lambda-A_{0}\right)^{-1}
$$

for all $\lambda>0$. Since, by Theorem $6, \lim _{t \rightarrow \infty} \mathrm{e}^{t A_{0}} f=P_{p, q} f$, Theorem 9 is a direct consequence of Theorem 8 and Lemma 3.

4.4. The semigroups generated by $\overline{\mathfrak{A}_{\varepsilon}}$

The space $C(\Omega)$ may be seen as the injective tensor product of the spaces $C(\mathcal{B})$ and $C(U)$ :

$$
C(\Omega)=C(\mathcal{B}) \tilde{\otimes}_{\epsilon} C(U),
$$

see, e.g., [79] pp. 45-50. This means that the supremum norm in $C(U)$ coincides with the injective norm inherited from $C(\mathcal{B})$ and $C(U)$, and the set of simple tensors, i.e., of functions of the form $f \otimes g, f \in C(\mathcal{B}) \times C(U)$ given by $(f \otimes g)(x, y, z)=$ $f(x, y) g(z),(x, y, z) \in \Omega$, is linearly dense in $C(\Omega)$. This allows constructing semigroups of operators in $C(\Omega)$ from building blocks available in $C(\mathcal{B})$ and $C(U)$ (see [63] pp. 21-24), as follows.

Since $\partial \mathcal{B}$ is assumed to be of class $C^{2, \kappa}, \kappa \in(0,1]$, the $2 D$ Laplace operator $\Delta_{2 D}$ with domain composed of $C^{2, \kappa}(\Omega)$ functions with normal derivatives vanishing on 
the boundary is closable, and its closure generates a conservative Feller semigroup in $C(\Omega)$ (see [36] p. 369). Let $\left(\mathrm{e}^{t \overline{\Delta_{2 D}}}\right)_{t \geq 0}$ be this semigroup, and let $\left(\mathrm{e}^{t A^{\varepsilon}}\right)_{t \geq 0}, \varepsilon \in(0,1]$ be the semigroups generated by $A^{\varepsilon}$ of (4.24).

For any $\varepsilon \in(0,1]$ and $t \geq 0$, one may think of the following map defined on the set of simple tensors

$$
f \otimes g \mapsto\left(\mathrm{e}^{t \overline{\Delta_{2 D}}} f\right) \otimes\left(\mathrm{e}^{t A^{\varepsilon}} g\right)
$$

Since such tensors form a linearly dense set in $C(\Omega)$, and since the supremum norm in $C(\Omega)$ coincides with the injective tensor norm inherited from $C(U)$ and $C(\mathcal{B})([79]$ pp. 49-50), this map may be extended to a bounded linear operator, say $\mathcal{T}_{\varepsilon}(t)$, in $C(\Omega)$ with norm 1 . This operator is positive and $\mathcal{T}_{\varepsilon}(t) 1_{\Omega}=1_{\Omega}$.

In [63] pp. 21-24, it is shown that so-constructed $\left(\mathcal{T}_{\varepsilon}(t)\right)_{t \geq 0}$ is a strongly continuous semigroup; this semigroup is termed the injective tensor product of semigroups $\left(\mathrm{e}^{t \overline{\Delta_{2 D}}}\right)_{t \geq 0}$ and $\left(\mathrm{e}^{t A^{\varepsilon}}\right)_{t \geq 0}$, and denoted

$$
\mathcal{T}_{\varepsilon}(t)=\mathrm{e}^{t \overline{\Delta_{2 D}}} \tilde{\otimes}_{\epsilon} \mathrm{e}^{t A^{\varepsilon}}
$$

Moreover, the set of linear combinations of simple tensors of the form $f \otimes g, f \in$ $D\left(\overline{\Delta_{2 D}}\right), g \in D\left(A^{\varepsilon}\right)$ is a core for the generator of this semigroup. It is clear that the last statement is also true if instead of $f \in D\left(\overline{\Delta_{2 D}}\right)$ one considers $f \in D\left(\Delta_{2 D}\right)$, and that $\left(\mathcal{T}_{\varepsilon}(t)\right)_{t \geq 0}$ is a conservative Feller semigroup.

Proposition 4. For any $\varepsilon \in(0,1]$, the operator $\mathfrak{A}_{\varepsilon}$ of $(3.2)$ is closable and its closure generates the semigroup $\left(\mathcal{T}_{\varepsilon}(t)\right)_{t \geq 0}$.

Proof. Arguing as in [17] p. 17 we conclude that at $z=0+$ and $z=0-, \partial_{z} u$ vanishes for $u \in D\left(\mathfrak{A}_{\varepsilon}\right)$, and this in turn implies that $\mathfrak{A}_{\varepsilon}$ satisfies the maximum principle.

For the sake of this proof, let $\mathcal{D}$ be the set of linear combinations of simple tensors of the form $f \otimes g, f \in D\left(\Delta_{2 D}\right), g \in D\left(A^{\varepsilon}\right)$, and let $\mathcal{A}_{\varepsilon}$ be the generator of the semigroup $\left(\mathcal{T}_{\varepsilon}(t)\right)_{t \geq 0}$. For a simple tensor $u=f \otimes g \in \mathcal{D}$

$$
\mathcal{A}_{\varepsilon} u=\left(\Delta_{2 D} f\right) \otimes g+f \otimes\left(A^{\varepsilon} g\right)=\left(\partial_{x}^{2}+\partial_{y}^{2}+\varepsilon^{-2} \partial_{z}^{2}\right) u
$$

(see [63] p. 23). Since it is clear that such a $u$ belongs also to $D\left(\mathfrak{A}_{\varepsilon}\right)$, the operators $\mathfrak{A}_{\varepsilon}$ and $\mathcal{A}_{\varepsilon}$ have the common subdomain $\mathcal{D}$ where they coincide. Next, for any $\lambda>0$, the range of $\left(\lambda-\mathcal{A}_{\varepsilon}\right)_{\mid \mathcal{D}}$ is dense in $C(\Omega)$, because $\mathcal{D}$ is a core for $\mathcal{A}_{\varepsilon}$ (see [36] Proposition 3.1 , p. 17). Therefore, also the range of $\lambda-\mathfrak{A}_{\varepsilon}$ is dense in $C(\Omega)$, since it contains $\left(\lambda-\mathfrak{A}_{\varepsilon}\right)_{\mid \mathcal{D}}=\left(\lambda-\mathcal{A}_{\varepsilon}\right)_{\mid \mathcal{D}}$. Thus, the operator $\mathfrak{A}_{\varepsilon}$, being clearly densely defined, is closable and its closure is a conservative Feller generator by Theorem 2.2. in [36] p. 165. Also, by the other implication in [36] Proposition 3.1, p. 17 just alluded to, $\mathcal{D}$ is a core for $\overline{\mathfrak{A}_{\varepsilon}}$. Hence, $\mathcal{D}$ being a common core for $\mathcal{A}_{\varepsilon}$ and $\overline{\mathfrak{A}_{\varepsilon}}$, these two generators must coincide. 
The subspace $C^{b}(\Omega)$ of Sect. 3 may be considered as an injective tensor product, too. Namely,

$$
C^{b}(\Omega)=C(\mathcal{B}) \tilde{\otimes}_{\epsilon} C(\{0-\} \cup\{0+\}) .
$$

where $C(\{0-\} \cup\{0+\})$, the space of continuous functions on the set $\{0-\} \cup\{0+\}$ with discrete topology may be identified with $\mathbb{R}^{2}$ with the maximum norm. Therefore, one may think of the injective tensor product semigroup $(\mathcal{S}(t))_{t \geq 0}$, where

$$
\mathcal{S}(t):=\mathrm{e}^{t \overline{\Delta_{2 D}}} \tilde{\otimes}_{\epsilon} \mathrm{e}^{t B}
$$

and $B$ was defined in Theorem 9 .

Proposition 5. The operator $\mathfrak{B}$ defined in (3.5) is the generator of the injective tensor product semigroup $(\mathcal{S}(t))_{t \geq 0}$.

Proof. It will be convenient to identify elements $g \in C(\{0-\} \cup\{0+\})$ with pairs of real numbers written as $\left(\begin{array}{c}g^{-} \\ g^{+}\end{array}\right)$. With this identification, a member $u$ of $C^{b}(\Omega)$ has the form

$$
u=u^{-} \otimes\left(\begin{array}{l}
1 \\
0
\end{array}\right)+u^{+} \otimes\left(\begin{array}{l}
0 \\
1
\end{array}\right),
$$

where $u^{-}$and $u^{+}$are defined in (3.4).

Let, for the sake of this proof, $\mathcal{B}$ be the generator of $(\mathcal{S}(t))_{t \geq 0}$. If $u$ is a member of $D(\mathfrak{B})$, i.e., if $u^{-}$and $u^{+}$belong to $D\left(\overline{\Delta_{2 D}}\right)$ then (by the already cited result from $\mathrm{p}$. 23 in [63]) (4.25) shows that $u \in D(\mathcal{B})$, and

$$
\begin{aligned}
\mathcal{B} u & =\overline{\Delta_{2 D}} u^{-} \otimes\left(\begin{array}{l}
1 \\
0
\end{array}\right)+u^{-} \otimes B\left(\begin{array}{l}
1 \\
0
\end{array}\right)+\overline{\Delta_{2 D}} u^{+} \otimes\left(\begin{array}{l}
0 \\
1
\end{array}\right)+u^{+} \otimes B\left(\begin{array}{l}
0 \\
1
\end{array}\right) \\
& =\overline{\Delta_{2 D}} u^{-} \otimes\left(\begin{array}{l}
1 \\
0
\end{array}\right)+u^{-} \otimes\left(\begin{array}{c}
-\alpha \\
\beta
\end{array}\right)+\overline{\Delta_{2 D}} u^{+} \otimes\left(\begin{array}{l}
0 \\
1
\end{array}\right)+u^{+} \otimes\left(\begin{array}{c}
\alpha \\
-\beta
\end{array}\right) \\
& =\left(\overline{\Delta_{2} D} u^{-}-\alpha u^{-}+\alpha u^{+}\right) \otimes\left(\begin{array}{l}
1 \\
0
\end{array}\right)+\left(\overline{\Delta_{2 D}} u^{+}+\beta u^{-}-\beta u^{+}\right) \otimes\left(\begin{array}{l}
0 \\
1
\end{array}\right) \\
& =\mathfrak{B} u .
\end{aligned}
$$

It follows that $\mathcal{B}$ extends $\mathfrak{B}$. However, since both $\mathcal{B}$ and $\mathfrak{B}$ are generators, $\mathcal{B}$ cannot be a proper extension of $\mathfrak{B}$ and we conclude that $\mathfrak{B}=\mathcal{B}$.

Proof of Theorem 3. Since simple tensors form a linearly dense subset of $C(\Omega)$ it suffices to show (3.6) for $u=f \otimes g$ where $f \in C(\mathcal{B})$ and $g \in C(U)$. By Theorem 9,

$$
\lim _{\varepsilon \rightarrow 0} \mathrm{e}^{t \overline{\mathfrak{A}_{\varepsilon}}}(f \otimes g)=\left(\mathrm{e}^{t \overline{\Delta_{2 D}}} f\right) \otimes\left(\lim _{\varepsilon \rightarrow 0} \mathrm{e}^{t A^{\varepsilon}} g\right)=\left(\mathrm{e}^{t \overline{\Delta_{2 D}}} f\right) \otimes\left(\mathrm{e}^{t B} P_{p, q} g\right), \quad t>0 .
$$

Since, as a direct calculation shows, $\mathcal{P}_{p, q}(f \otimes g)=f \otimes P_{p, q} g$, we have, on the other hand,

$$
\mathrm{e}^{t \mathfrak{B}} \mathcal{P}_{p, q}(f \otimes g)=\left(\mathrm{e}^{t \overline{\Delta_{2 D}}} f\right) \otimes\left(\mathrm{e}^{t B} P_{p, q} g\right),
$$

and this completes the proof. 


\section{Acknowledgements}

I am very grateful to an anonymous referee for careful reading of my paper and for a number of detailed and insightful comments that allowed me to improve the presentation and to enrich the bibliography.

Open Access. This article is licensed under a Creative Commons Attribution 4.0 International License, which permits use, sharing, adaptation, distribution and reproduction in any medium or format, as long as you give appropriate credit to the original author(s) and the source, provide a link to the Creative Commons licence, and indicate if changes were made. The images or other third party material in this article are included in the article's Creative Commons licence, unless indicated otherwise in a credit line to the material. If material is not included in the article's Creative Commons licence and your intended use is not permitted by statutory regulation or exceeds the permitted use, you will need to obtain permission directly from the copyright holder. To view a copy of this licence, visit http://creativecommons.org/licenses/ by $/ 4.0 /$.

Publisher's Note Springer Nature remains neutral with regard to jurisdictional claims in published maps and institutional affiliations.

\section{Appendix: Proof of Proposition 1}

As remarked by an anonymous referee of this paper, the following proof could be significantly simplified by invoking known perturbation results for quadratic forms, e.g., [61, Proposition 2.7] (with $j=I d$ and $S: H^{1}(\Omega) \rightarrow L^{2}(\partial \Omega)$ ) or [6, Proposition 4.4]. For the sake of the readers who, as myself, are less familiar with the theory of forms, I decided to keep the following more expanded and detailed version.

It is clear that $\mathfrak{H}$ is dense in $L^{2}(\Omega)$. Let (only in this proof)

$$
\mathfrak{b}_{\varepsilon}[u, v]=\int_{\Omega}\left[\partial_{x} u \partial_{x} \bar{v}+\partial_{y} u \partial_{y} \bar{v}+\varepsilon^{-2} \partial_{z} u \partial_{z} \bar{v}\right](x, y, z) \mathrm{d}(x, y, z), \quad u, v \in \mathfrak{H}
$$

and $\mathfrak{c}=\mathfrak{a}_{\varepsilon}-\mathfrak{b}_{\varepsilon}$ (note that $\mathfrak{c}$ does not depend on $\varepsilon$ ). Then, $\mathfrak{b}_{\varepsilon}$ is symmetric and, since $\varepsilon \in(0,1]$,

$$
\|\nabla u\|_{L^{2}(\Omega)}^{2}=\mathfrak{b}_{1}[u] \leq \mathfrak{b}_{\varepsilon}[u] \leq \varepsilon^{-2}\|\nabla u\|_{L^{2}(\Omega)}^{2} .
$$

It follows that the forms $\mathfrak{b}_{\varepsilon}$ are accretive. They are also closed, since using this inequality it may be shown that for each $\varepsilon$, the norm induced by $\mathfrak{b}_{\varepsilon}$ is equivalent to the norm in $\mathfrak{H}$.

Turning to analysis of $\mathfrak{c}$, we note first of all that it is bounded: there is a constant $C$ such that

$$
|\mathfrak{c}[u, v]| \leq C\|u\|_{\mathfrak{H}}\|v\|_{\mathfrak{H}}
$$

this is because all the trace operators (2.12) are bounded and $c^{-}, c^{+}, \alpha$ and $\beta$ are essentially bounded functions. Moreover, since the boundary of $\mathcal{B}$ is assumed to be Lipschitz continuous, all the trace operators (2.12) are compact (see [64] Thm 6.2, p. 
103). Hence, if a sequence $\left(u_{n}\right)_{n \geq 1}$ of elements of $\mathfrak{H}$ converges to 0 weakly, sequences of its traces converge strongly to zero in the corresponding $L^{2}$ spaces. Since $c^{+}, c^{-}, \alpha$ and $\beta$ are essentially bounded, it follows that $\lim _{n \rightarrow \infty} \mathfrak{c}\left[u_{n}\right]=0$. Hence, by Lemma 7.3 in [30], for each $\delta>0$ there exists a $c(\delta)>0$ such that

$$
|\mathfrak{c}[u]| \leq \delta\|u\|_{\mathfrak{H}}^{2}+c(\delta)\|u\|_{L^{2}(\Omega)}^{2} .
$$

By Theorem VI.3.11 in [53], this inequality combined with the fact that $\mathfrak{b}_{\varepsilon}$ is closed, shows that so is $\mathfrak{a}_{\varepsilon}=\mathfrak{b}_{\varepsilon}+\mathfrak{c}$. Moreover, taking $\delta=\frac{1}{2}$ in (4.26) we obtain, for $\gamma=2 c\left(\frac{1}{2}\right)+1$,

$$
\max \{|\Re \mathfrak{c}[u]|,|\mathfrak{s c}[u]|\} \leq \frac{1}{2} \mathfrak{b}_{1}[u]+\frac{\gamma}{2}\|u\|_{L^{2}(\Omega)}^{2} \leq \frac{1}{2} \mathfrak{b}_{\varepsilon}[u]+\frac{\gamma}{2}\|u\|_{L^{2}(\Omega)}^{2} .
$$

Thus,

$$
\left|\mathfrak{I a}_{\varepsilon}[u]\right|=|\mathfrak{I c}[u]| \leq \frac{1}{2} \mathfrak{b}_{\varepsilon}[u]+\frac{\gamma}{2}\|u\|_{L^{2}(\Omega)}^{2}
$$

and

$$
\Re \mathfrak{a}_{\varepsilon}[u] \geq \mathfrak{b}_{\varepsilon}[u]-|\mathfrak{R} \mathfrak{c}[u]| \geq \frac{1}{2} \mathfrak{b}_{\varepsilon}[u]-\frac{\gamma}{2}\|u\|_{L^{2}(\Omega)}^{2}
$$

It follows that

$$
\left|\mathfrak{s} \mathfrak{a}_{\varepsilon}[u]\right| \leq \Re \mathfrak{a}_{\varepsilon}[u]+\gamma\|u\|_{L^{2}(\Omega)}^{2} .
$$

Since $\Im\left(\mathfrak{a}_{\varepsilon}+\gamma\right)[u]=\Im \mathfrak{a}_{\varepsilon}[u]$ and $\gamma[u]=\gamma\|u\|_{L^{2}(\Omega)}^{2}$, this inequality is equivalent to (2.16).

\section{REFERENCES}

[1] R. A. Adams and J. J. F. Fournier, Sobolev Spaces, Second edition, Pure and Applied Mathematics (Amsterdam), vol. 140, Elsevier, Amsterdam, 2003.

[2] S. S. Andrews, Accurate particle-based simulation of adsorption, desorption and partial transmission, Phys. Biol. 6 (2010), 046015.

[3] W. Arendt,Semigroups and Evolution Equations: Functional Calculus, Regularity and Kernel Estimates, Evolutionary Equations vol. 1, 2004, pp. 1-85.

[4] W. Arendt, Heat Kernels - Manuscript of the 9th Internet Seminar, 2006. Freely available at http://www.uni-ulm.de/fileadmin/website_uni_ulm/mawi.inst.020/arendt/downloads/ internetseminar.pdf.

[5] W. Arendt, C. J. K. Batty, M. Hieber, and F. Neubrander, Vector-Valued Laplace Transforms and Cauchy Problems, Birkhäuser, Basel, 2001.

[6] W. Arendt, A.F.M. ter Elst, J.B. Kennedy, and M. Sauter, The Dirichlet-to-Neumann operator via hidden compactness, Journal of Functional Analysis 266 (2014), no. 3, 1757-1786.

[7] J. M. Arrieta, A. N. Carvalho, M. C. Pereira, and R. P. Silva, Semilinear parabolic problems in thin domains with a highly oscillatory boundary, Nonlinear Anal. 74 (2011), no. 15, 5111-5132.

[8] C. Bardos, D. Grebenkov, and A. Rozanova-Pierrat, Short-time heat diffusion in compact domains with discontinuous transmission boundary conditions, Math. Models Methods Appl. Sci. 26 (2016), no. 1, 59-110. 
[9] S. R. M. Barros and M. C. Pereira, Semilinear elliptic equations in thin domains with reaction terms concentrating on boundary, J. Math. Anal. Appl. 441 (2016), no. 1, 375-392.

[10] A. Bobrowski, Degenerate convergence of semigroups, Semigroup Forum 49 (1994), no. 3, 303327.

[11] A. Bobrowski, Functional Analysis for Probability and Stochastic Processes. An Introduction, Cambridge University Press, Cambridge, 2005.

[12] A. Bobrowski, On a semigroup generated by a convex combination of two Feller generators, J. Evol. Equ. 7 (2007), no. 3, 555-565.

[13] A. Bobrowski, Generation of cosine families via Lord Kelvin's method of images, J. Evol. Equ. 10 (2010), no. 3, 663-675.

[14] A. Bobrowski, Lord Kelvin's method of images in the semigroup theory, Semigroup Forum 81 (2010), 435-445.

[15] A. Bobrowski, From diffusions on graphs to Markov chains via asymptotic state lumping, Ann. Henri Poincare 13 (2012), 1501-1510.

[16] A. Bobrowski, Singular perturbations involving fast diffusion, J. Math. Anal. Appl. 427 (2015), no. 2, 1004-1026.

[17] A. Bobrowski, Convergence of One-parameter Operator Semigroups. In Models of Mathematical Biology and Elsewhere, New Mathematical Monographs, vol. 30, Cambridge University Press, Cambridge, 2016.

[18] A. Bobrowski, Generators of Markov Chains. From a Walk in the Interior to a Dance on the Boundary, Cambridge University Press, Cambridge, 2020.

[19] A. Bobrowski, Modeling diffusion in thin 2D layers separated by a semi-permeable membrane, SIAM Journal on Mathematical Analysis 52 (2020), no. 4, 3222-3251, available at https://doi.org/ 10.1137/19M1291443.

[20] A. Bobrowski, A. Gregosiewicz, and M. Murat, Functionals-preserving cosine families generated by Laplace operators in C[0,1], Discr. Cont. Dyn. Syst. B 20 (2015), no. 7, 1877-1895.

[21] A. Bobrowski, B. Kaźmierczak, and M. Kunze, An averaging principle for fast diffusions in domains separated by semi-permeable membranes, Mathematical Models and Methods in Applied Sciences 27 (2017), no. 04, 663-706, available athttp://www.worldscientific.com/doi/pdf/10.1142/ S0218202517500130.

[22] A. Bobrowski and M. Kunze, Irregular convergence of mild solutions of semilinear equations, J. Math. Anal. Appl. 472 (2019), no. 2, 1401-1419.

[23] A. Bobrowski and T. Lipniacki, Singular limit of diffusion equations in 3D domains with thickness converging to zero, Models and Reality: Festschrift For James Robert Thompson, edited by J.A. Dobelman, 2017, pp. 95-116.

[24] A. Bobrowski and T. Lipniacki, Robin-type boundary conditions in transition from reactiondiffusion equations in $3 D$ domains to equations in $2 D$ domains, Journal of Differential Equations 268 (2019), 239-271.

[25] A. Bobrowski and K. Morawska, From a PDE model to an ODE model of dynamics of synaptic depression, Discr. Cont. Dyn. Syst. B 17 (2012), no. 7, 2313-2327.

[26] Z. Brzeźniak, G. Dhariwal, and Q. T. Le Gia, Stochastic Navier-Stokes equations on a thin spherical domain, ArXiv e-prints (2020), available at arXiv:2002.08873v2.

[27] T. Carlsson, T. Ekholm, and C. Elvingson, Algorithm for generating a Brownian motion on a sphere, Journal of Physics A: Mathematical and Theoretical 43 (2010), no. 50, 505001.

[28] C. Costantini and T.G. Kurtz, Existence and uniqueness of re ecting diffusions in cusps, Electron. J. Probab. 23 (2018), Paper No. 84, 21.

[29] J. Crank, The mathematics of diffusion, Second Edition, Clarendon Press, Oxford, 1975.

[30] D. Daners, Principal eigenvalues for generalised indefinite Robin problems, Potential Anal. 38 (2013), no. 4, 1047-1069. MR3042694.

[31] M. H. A. Davis, Lectures on Stochastic Control and Nonlinear Filtering, Springer, 1984.

[32] M. H. A. Davis, Piece-wise Deterministic Markov Processes, J. Royal Statistical Soc., Ser. B. 46 (1984), 353-388.

[33] M. H. A. Davis,Markov Processes and Optimization, Chapman and Hall, 1993.

[34] T. Elsken, Continuity of attractors for net-shaped thin domains, Topol. Methods Nonlinear Anal. 26 (2005), no. 2, 315-354. 
[35] K.-J. Engel and R. Nagel, One-Parameter Semigroups for Linear Evolution Equations, Springer, New York, 2000.

[36] S. N. Ethier and T. G. Kurtz, Markov Processes. Characterization and Convergence, Wiley, New York, 1986.

[37] A. Favini, G. R. Goldstein, J. A. Goldstein, and S. Romanelli, The one-dimensional wave equation with Wentzell boundary conditions, Differential Equations and Control Theory (Athens, OH, 2000), 2002, pp. 139-145.

[38] W. Feller, Diffusion processes in one dimension, Trans. Amer. Math. Soc. 77 (1954), no. 1, 1-31.

[39] W. Feller, An Introduction to Probability Theory and Its Applications, Vol. 2, Wiley, New York, 1966. Second edition, 1971.

[40] E. Fieremans, D. S Novikov, J. H. Jensen, and J. A. Helpern, Monte Carlo study of a twocompartment exchange model of diffusion, NMR in Biomedicine 23 (2010), 711- 724.

[41] P. B. Gilkey and K. Kirsten, Heat content asymptotics with transmittal and transmission boundary conditions, Journal of the London Mathematical Society 68 (2003), no. 2, 431-443, available at https://londmathsoc.onlinelibrary.wiley.com/doi/pdf/10.1112/S0024610703004526.

[42] J. A. Goldstein, Semigroups of Linear Operators and Applications, Oxford University Press, New York, 1985

[43] G. Greiner, Perturbing the boundary conditions of a generator, Houston J. Math. 13 (1987), no. 2, 213-229.

[44] R. J. Griego and R. Hersh, Random evolutions, Markov chains, and systems of partial differential equations, Proc. Nat. Acad. Sci. U.S.A. 62 (1969), 305-308.

[45] R. J. Griego and R. Hersh, Theory of random evolutions with applications to partial differential equations, Trans. Amer. Math. Soc. 156 (1971), 405-418.

[46] J. K. Hale and G. Raugel, Reaction-diffusion equation on thin domains, J. Math. Pures Appl. (9) 71 (1992), no. 1, 33-95.

[47] B. Hat, B. Kaźmierczak, and T. Lipniacki, B cell activation triggered by the formation of the small receptor cluster: a computational study, PLoS Comput Biol. 7(10) (2011 Oct.), e1002197.

[48] B. Hat, P. Paszek, M. Kimmel, K. Piechór, and T. Lipniacki, How the number of alleles influences gene expression, J. Statist. Phys. 128 (2007), no. 1/2, 511-533.

[49] A. M. Il'in, R. Z. Khasminskii, and G. Yin, Asymptotic expansions of solutions of integro-differential equations for transition densities of singularly perturbed switching diffusions: rapid switchings, J. Math. Anal. Appl. 238 (1999), 516-539.

[50] K. Itô and McKean, Jr. H. P., Diffusion Processes and Their Sample Paths, Springer, Berlin, 1996. Repr. of the 1974 ed.

[51] O. Kallenberg, Foundations of Modern Probability, 2nd ed., Springer, 2002.

[52] I. Karatzas and S. E. Shreve, Brownian Motion and Stochastic Calculus, Springer, New York, 1991.

[53] T. Kato, Perturbation Theory for Linear Operators, Classics in Mathematics Series, Springer, 1995. reprint of the 1980 edition.

[54] B. Kaźmierczak and T. Lipniacki, Regulation of kinase activity by diffusion and feedback, J. Theor. Biol. 259 (2009), 291-296.

[55] T. G. Kurtz, Extensions of Trotter's operator semigroup approximation theorems, J. Functional Analysis 3 (1969), 354-375.

[56] T. G. Kurtz, A limit theorem for perturbed operator semigroups with applications to random evolutions, J. Functional Analysis 12 (1973), 55-67.

[57] T. G. Kurtz, Applications of an abstract perturbation theorem to ordinary differential equations, Houston J. Math. 3 (1977), no. 1, 67-82.

[58] T. G. Kurtz, A control formulation for constrained Markov processes, Mathematics of random media (Blacksburg, VA, 1989), 1991, pp. 139-150.

[59] A. Lejay, The snapping out Brownian motion, Ann. Appl. Probab. 26 (2016), no. 3, 1727-1742.

[60] T. M. Liggett, Continuous Time Markov Processes. An Introduction, Amer. Math. Soc., 2010.

[61] D. Mugnolo and R. Nittka, Convergence of operator semigroups associated with generalized elliptic forms, J. Evol. Equ. 12 (2012), 593-619.

[62] D. Mugnolo, R. Nittka, and O. Post, Norm convergence of sectorial operators on varying Hilbert spaces, Oper. Matrices 7 (2013), no. 4, 955-995. 
[63] R. Nagel (ed.), One-parameter Semigroups of Positive Operators, Lecture Notes in Mathematics, vol. 1184, Springer, 1986.

[64] J. Nečas, Direct methods in the theory of elliptic equations, Springer Monographs in Mathematics, Springer, Heidelberg, 2012.

[65] G. Nickel, A new look at boundary perturbations of generators, Electron. J. Differential Equations (2004), No. 95, 14.

[66] E. M. Ouhabaz, Second order elliptic operators with essential spectrum [0;1) on Lp, Comm. Partial Differential Equations 20 (1995), no. 5-6, 763-773.

[67] E. M. Ouhabaz, Analysis of Heat Equations on Domains, Lond. Math. Soc. Monograph Series, vol. 30, Princeton Univ. Press, Princeton, 2005.

[68] I. Pažanin and M. C. Pereira, On the nonlinear convection-diffusion-reaction problem in a thin domain with a weak boundary absorption, Commun. Pure Appl. Anal. 17 (2018), no. 2, 579-592.

[69] A. Pazy, Semigroups of Linear Operators and Applications to Partial Differential Equations, Springer, 1983.

[70] M. A. Pinsky, Lectures on Random Evolutions, World Scientific, Singapore, 1991.

[71] A. Posilicano, Self-adjoint extensions of restrictions, Oper. Matrices 2 (2008), no. 4, 483-506.

[72] O. Post, Spectral analysis on graph-like spaces, Lecture Notes in Mathematics, vol. 2039, Springer, Heidelberg, 2012.

[73] J. G. Powles, M. J. D. Mallett, G. Rickayzen, and W. A. B. Evans, Exact analytic solutions for diffusion impeded by an infinite array of partially permeable barriers, Proc. Roy. Soc. London Ser. A 436 (1992), no. 1897, 391-403.

[74] M. Prizzi, M. Rinaldi, and K. P. Rybakowski, Curved thin domains and parabolic equations, Studia Math. 151 (2002), no. 2, 109-140.

[75] M. Prizzi and K. P. Rybakowski, The effect of domain squeezing upon the dynamics of reactiondiffusion equations, J. Differential Equations 173 (2001), no. 2, 271-320.

[76] M. Prizzi and K. P. Rybakowski, Recent results on thin domain problems. II, Topol. Methods Nonlinear Anal. 19 (2002), no. 2, 199-219.

[77] G. Raugel, Dynamics of partial differential equations on thin domains, Dynamical systems (Montecatini Terme, 1994), 1995, pp. 208-315. MR1374110

[78] R. Rudnicki and M. Tyran-Kamińska, Piecewise Deterministic Processes in Biological Models, Springer Briefs in Applied Sciences and Technology, Springer, Cham, 2017. Springer Briefs in Mathematical Methods.

[79] R. A. Ryan, Introduction to Tensor Products of Banach Spaces, Springer, 2002.

[80] B. Simon, A canonical decomposition for quadratic forms with applications to monotone convergence theorems, J. Functional Analysis 28 (1978), no. 3, 377-385.

[81] M. Sova, Convergence d'opérations linéaires non bornées, Rev. Roumaine Math. Pures Appl. 12 (1967), 373-389.

[82] J. E. Tanner, Transient diffusion in a system partitioned by permeable barriers. Application to NMR measurements with a pulsed field gradient, The Journal of Chemical Physics 69 (1978), no. 4, 1748-1754.

[83] T.-J. Xiao and J. Liang, A solution to an open problem for wave equations with generalized Wentzell boundary conditions, Math. Ann. 327 (2003), no. 2, 351-363.

[84] T.-J. Xiao and J. Liang, Second order differential operators with Feller-Wentzell type boundary conditions, J. Funct. Anal. 254 (2008), no. 6, 1467-1486.

[85] G. Yin, On limit results for a class of singularly perturbed switching diffusions, J. Theor. Probab. 14 (2001), 673-697.

[86] G. Yin and M. Kniazeva, Singularly perturbed multidimensional switching diffusions with fast and slow switchings, J. Math. Anal. Appl. 229 (1999), 605-630. 


\author{
Adam Bobrowski \\ Lublin University of Technology \\ Nadbystrzycka $38 \mathrm{~A}$ \\ 20-618 Lublin \\ Poland \\ E-mail: a.bobrowski@pollub.pl
}

Accepted: 4 September 2020 